\title{
From quarks and gluons to color superconductivity at supranuclear densities
}

\author{
Jens Braun ${ }^{1,2}$ and Benedikt Schallmo ${ }^{1}$ \\ ${ }^{1}$ Institut für Kernphysik, Technische Universität Darmstadt, D-64289 Darmstadt, Germany \\ ${ }^{2}$ ExtreMe Matter Institute EMMI, GSI, Planckstraße 1, D-64291 Darmstadt, Germany
}

(Received 17 August 2021; revised 17 November 2021; accepted 13 December 2021; published 7 February 2022)

\begin{abstract}
We study the emergence of color superconductivity in the theory of the strong interaction at supranuclear densities. To this end, we follow the renormalization group (RG) flow of dense strong-interaction matter with two massless quark flavors from the fundamental quark and gluon degrees of freedom at high energies down to the nonperturbative low-energy regime which is found to be governed by the dynamical formation of diquark states. With the strong coupling at the initial RG scale as the only input parameter, we compute the (chirally symmetric) scalar diquark condensate and analyze its scaling behavior over a wide range of the quark chemical potential. Approximations entering our computations are critically assessed. Since our approach naturally allows us to study the scale dependence of couplings, we also monitor the strength of couplings appearing in low-energy models of dense strong-interaction matter. The observed dependence of these couplings on the quark chemical potential may help to amend model studies in the future. Finally, we estimate the speed of sound of dense QCD matter. Our results indicate that the speed of sound exceeds the value of the noninteracting quark gas at high densities and even increases as the density is decreased, across a wide range, suggesting the existence of a maximum at supranuclear densities.
\end{abstract}

DOI: $10.1103 /$ PhysRevD.105.036003

\section{INTRODUCTION}

There is interest in the properties of quantum chromodynamics (QCD) at supranuclear densities ever since the first discussion of the possible existence of colorsuperconducting ground states in the 1970s, see Ref. [1] for an early review. However, the properties of such states remained elusive for a long time. In the late 1990s, it was then found that the formation of sizeable pairing gaps in color-superconducting phases may considerably affect the dynamics of QCD at low temperatures, see Refs. [2-11] for reviews.

More recently, the interest in the properties of dense strong-interaction matter received a significant boost because of the first detection of the gravitational-wave signal of a neutron-star merger [12,13], ongoing missions aiming at first direct neutron-star radius measurements [14-19], as well as precise mass measurements of heavy neutron stars [20-23]. These breakthroughs provide important constraints for the equation of state (EOS) of stronginteraction matter, see Ref. [24] for a recent analysis. Quantitative theoretical results for the EOS of dense

Published by the American Physical Society under the terms of the Creative Commons Attribution 4.0 International license. Further distribution of this work must maintain attribution to the author(s) and the published article's title, journal citation, and DOI. Funded by SCOAP . strong-interaction matter are therefore indeed urgently needed in view of this tremendous progress made in the observation of neutron stars. In addition, constraints on the EOS can be obtained from heavy-ion collisions [25]. Nevertheless, a reliable description of the properties and dynamics of strong-interaction matter over a wide range of densities and temperatures still represents a formidable challenge, from an observational, experimental, and theoretical standpoint.

Presently, studies based on chiral effective field theory (EFT) interactions (see, e.g., Ref. [26] for a review) set benchmarks and yield strong constraints for the EOS in the low-density regime [27,28], see Ref. [29] for a recent review. For low to moderate densities, functional renormalization group (fRG) studies of nucleon-meson [30-32] and quark-meson models [33-35] aiming at the EOS at low temperatures are also available. At very high density, constraints for the EOS come from perturbative QCD (pQCD) studies [36-42]. However, in the broad intermediate density regime, where both the chiral and the pQCD expansion are expected to break down, much less is known about the dynamical degrees of freedom and their interactions, resulting in large uncertainties for the EOS and other quantities, such as the speed of sound of dense matter. In this density regime, which is still relevant for astrophysical applications, QCD is widely expected to be governed by a color-superconducting ground state (see Refs. [2-11] for reviews). 
Renormalization group (RG) approaches have played and are still playing an outstanding role in the analysis of the symmetry-breaking patterns and the emergence of color-superconducting ground states in dense matter [43-47]. Indeed, since systems of this kind represent a multi-scale problem, RG approaches are very well suited. Recently, an analysis of the RG flow of gluon-induced fourquark interaction channels in a Fierz-complete setting for two massless quark flavors has been performed to gain a deeper insight into symmetry breaking patterns underlying QCD over a wide range of densities at low and intermediate temperatures [47]. There, it was found that the scalarpseudoscalar interaction channel dominates the dynamics for small chemical potentials. Increasing the chemical potential, a (small) range of chemical potentials opens up with many interaction channels of roughly equal strength, indicating that the structure of the ground state may be very complicated in this regime. Increasing the chemical potential further, it was then observed that the diquark channel becomes most dominant, suggesting the formation of a chirally symmetric diquark condensate associated with pairing of the two-flavor color-superconductor (2SC) type. This observation is in accordance with early studies [48-51], including first-principles calculations which exploit the fact that the coupling effectively becomes small in the high-density limit owing to asymptotic freedom [43,44,52-56].

The RG analysis of the symmetry-breaking patterns in Ref. [47] laid the ground for a subsequent computation of constraints from quark-gluon dynamics for the EOS of isospin-symmetric two-flavor QCD over a wide range of densities [28]. Remarkably, toward the nucleonic lowdensity regime, the results from this EOS study are impressively consistent with those from calculations based on chiral EFT interactions. Moreover, the RG study of the EOS in Ref. [28] predicts the emergence of a maximum in the speed of sound at supranuclear densities which appears to be tightly connected to the formation of a diquark gap. Interestingly, this maximum exceeds the asymptotic highdensity value of the speed of sound. However, its exact position in terms of the density has not yet been determined conclusively. With respect to astrophysical applications, it is worth noting that the analysis of constraints from neutron-star masses also strongly suggests the existence of a maximum of the speed of sound for neutron-rich matter [24,57-60].

With our present work, we aim at laying the fieldtheoretical foundation for new first-principles studies of the EOS of dense QCD matter. As a first application, we shall demonstrate that - starting from the fundamental quark and gluon degrees of freedom at high energies-our RG approach allows us to study the dynamical formation of diquarks in the low-energy limit. In a next step, for example, this can be used to narrow down the (systematic) uncertainties of the thermodynamic quantities computed in
Ref. [28], in particular those of the EOS and the position of the maximum of the speed of sound. Still, the analysis of the RG flows presented in this work already allows us to gain an insight into the dynamics of dense QCD matter over a wide range of chemical potentials, as we shall show by computing the diquark gap.

The present work is organized as follows: In Sec. II, we discuss the formalism underlying our RG analysis of dense QCD matter. This includes a discussion of possible extensions required for computations of the EOS and also makes connections to our previous study of the EOS of dense matter [28]. The RG flow of dense QCD matter is then analyzed in detail in Sec. III. There, we also present our results for the (chirally symmetric) scalar diquark condensate as a function of the quark chemical potential. In Sec. IV, we finally discuss implications of our RG study for low-energy models of dense strong-interaction matter and for the speed of sound as a specific example for a phenomenologically important thermodynamic quantity. Our conclusions and a brief outlook can be found in Sec. V.

\section{FORMALISM}

\section{A. Effective action}

For our analysis of the properties of QCD at intermediate and also high densities, we employ the Wetterich equation [61] which is an RG equation for the quantum effective action $\Gamma$. Within this framework, the effective action depends on a so-called RG "time" $t=\ln (k / \Lambda)$ where $k$ is the RG scale and $\Lambda$ may be chosen to be the scale at which the initial condition $\Gamma_{k=\Lambda}$ for the scale-dependent effective action $\Gamma_{k}$ is fixed. In our present study, the initial condition is given by the classical (Euclidean) QCD action $S$ for two massless quark flavors coming in three colors:

$$
S=\int \mathrm{d}^{4} x\left\{\frac{1}{4} F_{\mu \nu}^{a} F_{\mu \nu}^{a}+\bar{\psi}\left(\mathrm{i} \not \partial+\bar{g} \not A-\mathrm{i} \gamma_{0} \mu\right) \psi\right\} .
$$

Here, $\bar{g}$ is the bare gauge coupling and $\mu$ is the quark chemical potential. For the values of $\mu$ considered in this work, we choose $\Lambda \gg \mu$ to ensure that the RG flow is initialized in the perturbative high-energy regime. The gluon fields $A_{\mu}^{a}$ come with Lorentz (greek letters) and color (roman letters) indices and enter the definition of the field-strength tensor $F_{\mu \nu}^{a}=\partial_{\mu} A_{\nu}^{a}-\partial_{\nu} A_{\mu}^{a}+\bar{g} f^{a b c} A_{\mu}^{b} A_{\nu}^{c}$ $(a=1, \ldots, 8)$. Moreover, they are coupled to the quark fields $\psi$ via the quark-gluon vertex, see Eq. (1). Note that the quark fields $\psi$ carry color and flavor components.

The quark-gluon vertex generates a plethora of interaction channels. With respect to studies of ground-state properties, quark self-interaction channels are of particular importance as they can be directly related to the orderparameter potential of QCD. More specifically, the quarkgluon vertex induces four-quark interactions already at the one-loop level via two-gluon exchange. Schematically, this 
leads to corrections of the effective action of the following form:

$$
\Delta \Gamma=\int \mathrm{d}^{4} x \sum_{i} \bar{\lambda}_{i}\left(\bar{\psi} \mathcal{O}_{i} \psi\right)^{2}
$$

Here, $\mathcal{O}_{i}$ determines the color, flavor, and Dirac structure of the four-quark vertex. Note that, in contrast to low-energy model studies, the four-quark couplings $\bar{\lambda}_{i}$ are not free parameters but generated from fundamental quark-gluon interactions, $\bar{\lambda}_{i} \sim \bar{g}^{4}$. Higher quark self-interactions are parametrically suppressed at high momentum scales. For example, eight quark-interactions scale as $\sim \bar{g}^{8}$. However, following the RG flow from high to low momentum scales, such higher-order interaction channels then become increasingly important. In fact, in regimes where the symmetry is broken spontaneously, eight quark interactions determine the masses of bound states of two quarks. We shall come back to this below. In particular, we shall discuss the relevance of eight-quark interactions at different scales in Sec. IV, which may also provide useful information for the construction of low-energy models at intermediate and high densities.

Still, already an analysis of the RG flow of gluoninduced four-quark interactions in the pointlike limit ("zero-momentum projection") can provide us with an important insight into the symmetry-breaking patterns over a wide range of temperatures and quark chemical potentials, see Ref. [62] for an introduction. In fact, this has been successfully demonstrated for QCD in the vacuum limit [63], at finite temperature [64,65], and over a wide range of chemical potentials [47]. In the latter study, it has been found within a Fierz-complete two-flavor setting that the scalar-pseudoscalar channel is most dominant at low densities, in accordance with full QCD RG-flows in the vacuum limit $[66,67]$. At large chemical potentials, which are at the heart of the present work, the diquark channel $\sim\left(\bar{\psi}_{b} \tau_{2} \epsilon_{a b c} \gamma_{5} \mathcal{C} \bar{\psi}_{c}^{T}\right)\left(\psi_{d}^{T} \mathcal{C} \gamma_{5} \tau_{2} \epsilon_{a d e} \psi_{e}\right)$ is then dynamically rendered the most dominant channel, suggesting the formation of a chirally symmetric diquark condensate associated with pairing of the two-flavor color-superconductor (2SC) type [47]. ${ }^{1}$ This is in accordance with early studies of dense QCD [44,48,49,51-53].

Although studies of the RG flow of four-quark interactions in the pointlike approximation provide a deep insight into symmetry-breaking patterns and their dependence on external control parameters, they are restricted to scales $k \geq k_{\mathrm{SB}}$, where the scale $k_{\mathrm{SB}}$ is associated with spontaneous symmetry breaking, such as chiral symmetry breaking or $\mathrm{U}(1)_{\mathrm{V}}$ symmetry breaking. In such a setting, symmetry breaking is indicated by a specific four-quark

\footnotetext{
${ }^{1}$ Here, $\tau_{2}$ is the second Pauli matrix and, in color space, it is summed over the totally antisymmetric tensor $\epsilon_{a b c}$. Moreover, we have introduced $\mathcal{C}=\mathrm{i} \gamma_{2} \gamma_{0}$.
}

channel approaching criticality associated with a divergence of the corresponding coupling at the scale $k_{\mathrm{SB}}$. Below this scale, the dynamics is governed by the formation of condensates. However, an analysis of the groundstate properties of QCD in this low-energy regime $k<k_{\mathrm{SB}}$ requires to go beyond the pointlike limit and to resolve the momentum dependences of the quark correlation functions. Indeed, information on bound-state and condensate formation is encoded in the momentum structure of the quark correlation functions. Such momentum dependences can be conveniently resolved by employing a HubbardStratonovich transformation of at least the most dominant four-quark interaction channel. For example, as demonstrated in Ref. [28], one may perform such a transformation of gluon-induced four-quark interactions at a given scale $\Lambda_{0}>k_{\mathrm{SB}}$, which then gives access to the low-energy regime. However, this introduces a dependence of the effective action on the scale $\Lambda_{0}$ which is reflected in an uncertainty for the results for low-energy observables, see Ref. [28] for a discussion in the context of dense QCD. The dependence on this artificial scale $\Lambda_{0}$ can be removed by employing the so-called dynamical hadronization technique [68-74], see also Ref. [75] for recent developments regarding the study of quark composites. Loosely speaking, this technique implements continuous Hubbard-Stratonovich transformations of four-quark interactions in the RG flow and thereby allows us to continuously follow the RG flow from the classical QCD action at high-momentum scales down to the deep infrared regime which is governed by the formation of bound states and condensates. We shall apply this technique in the following.

The present work should be viewed as the next step in a series of studies [28,47,76-78]. However, we do not aim at quantitative studies of thermodynamic quantities and lowenergy observables. We rather aim at setting the methodological stage for subsequent new quantitative computations in this series.

Let us now be specific and construct our ansatz for the scale-dependent effective action $\Gamma_{k}$ which underlies our present study of dense QCD matter. As in our previous works, see, e.g., Ref. [47], we rely on the background field approach to gauge theories $[79,80]$ within background covariant gauges and employ the background field approximation which has been worked out in detail for applications in perturbative as well as nonperturbative settings over many years by now, see, e.g., Refs. [81-90] and, for a recent detailed fRG review on this aspect, see Ref. [91]. In this approach, the so-called background field effective action inherits gauge invariance from gauge transformations of an auxiliary background field. The equivalence of this invariance with the actual physical gauge invariance follows from the on-shell background independence of this approach and the Slavnov-Taylor identities, where the background independence is encoded in Nielsen identities. With these identities, it can then be shown that the 
correlation functions associated with the background field are indeed related to elements of the $S$-matrix [92]. In fRG studies, however, the regulator functions for fields carrying a net color charge break gauge invariance explicitly and, as a consequence, the independence of the auxiliary background field is also lost. This eventually leads to modifications of the Slavnov-Taylor and the Nielsen identities. Note that the latter also monitor the difference of correlation functions associated with the background field and those associated with the fluctuation field. In general, the construction of a manifestly gauge-invariant effective action in the spirit of the background-field approach may therefore be nontrivial within the fRG framework. In the present work, we treat the gauge sector as developed and discussed in detail in Refs. [63,65,81,82,86]. More specifically, manifest gauge invariance of the solution in these studies is maintained by identifying the full gauge field with the background field in the RG flow. Thus, in the following, we assume that the background-field two-point function can be identified with the one of the fluctuation field in the flow, which is an approximation. For a treatment of the difference of these two quantities, we refer the reader to Ref. [93]. This approximation entails that the RG flow is no longer closed [94] and only some constraints imposed by the modified Slavnov-Taylor identities are satisfied. As in previous works $[63,65,81,82,86]$, we shall assume that corrections due to this approximation are subleading, which is at least reasonable in the (semi-)perturbative regime above the symmetry breaking scale $k_{\mathrm{SB}}$. A detailed discussion of these issues can be found in Ref. [91]. In any case, the advantage of our present approach is that it equips us with a gauge-invariant approximate solution of the effective action.

Since we would like to study the RG flow from the perturbative high-momentum regime down to the lowenergy regime governed by the formation of bound states of quarks, we basically employ a combination of the classical QCD action given in Eq. (1) and an ansatz for the low-energy sector associated with complex-valued scalar diquark fields $\Delta_{a}$ describing quark composites of the form $\sim\left(\psi_{b}^{T} \mathcal{C} \gamma_{5} \tau_{2} \epsilon_{a b c} \psi_{c}\right)$ :

$$
\begin{aligned}
\Gamma_{k}= & \int \mathrm{d}^{4} x\left\{\bar{\psi}_{b}\left(\mathrm{i} \gamma_{\mu} D_{\mu}^{b c}-\mathrm{i} \mu \gamma_{0}\right) \psi_{c}+Z_{\Delta}\left(D_{\mu}^{c a} \Delta_{a}\right)\left(D_{\mu}^{c b} \Delta_{b}\right)^{*}+2 \mu Z_{\Delta}\left(\Delta_{a}\left(D_{0}^{a b} \Delta_{b}\right)^{*}-\Delta_{a}^{*}\left(D_{0}^{a b} \Delta_{b}\right)\right)-4 \mu^{2} Z_{\Delta} \Delta_{a}^{*} \Delta_{a}\right. \\
& +\frac{1}{2} \bar{\lambda}_{\mathrm{csc}}\left(\bar{\psi}_{b} \tau_{2} \mathrm{i} \epsilon_{a b c} \gamma_{5} \mathcal{C} \bar{\psi}_{c}^{T}\right)\left(\psi_{d}^{T} \mathcal{C} \gamma_{5} \tau_{2} \mathrm{i} \epsilon_{a d e} \psi_{e}\right)+\frac{1}{2} \mathrm{i} \bar{h}\left(\psi_{b}^{T} \mathcal{C} \gamma_{5} \tau_{2} \Delta_{a} \epsilon_{a b c} \psi_{c}\right)-\frac{1}{2} \mathrm{i} \bar{h}\left(\bar{\psi}_{b} \gamma_{5} \tau_{2} \Delta_{a}^{*} \epsilon_{a b c} \mathcal{C} \bar{\psi}_{c}^{T}\right) \\
& \left.+\bar{m}^{2} \Delta_{a}^{*} \Delta_{a}+\bar{\lambda}_{\Delta}\left(\Delta_{a}^{*} \Delta_{a}\right)^{2}+\frac{1}{4} Z_{A} F_{\mu \nu}^{a} F_{\mu \nu}^{a}\right\}+\Delta \Gamma_{\mathrm{gf}}+\Delta \Gamma_{\mathrm{gh}} .
\end{aligned}
$$

Here, $D_{\mu}^{b c}=\partial_{\mu} \delta^{b c}-\mathrm{i} \bar{g} A_{\mu}^{a} T_{b c}^{a}$ and $a, b, c$ are color indices. We have suppressed flavor indices for readability. Note that we do not take into account the running of the wave function renormalization of the quark fields in our present exploratory study since it depends only mildly on the RG scale, at least at small densities $[66,67,69,73,74,95,96]$.

The diquark $\left(\Delta_{a}^{*}\right)$ /antidiquark $\left(\Delta_{a}\right)$ fields appearing in Eq. (3) transform as an antitriplet/triplet in color space. Note that we include only these fields as effective low-energy degrees of freedom. This is motivated by the fact that the diquark channel has been found to be the most dominant interaction channel for $\mu \gtrsim 350 \mathrm{MeV}$ in a Fierz-complete study of gluon-induced four-quark interaction channels [47]. Other four-quark channels, such as the scalar-pseudoscalar interaction channel associated with pion dynamics, have been found to be clearly subdominant in this regime, provided that the $\mathrm{U}(1)_{\mathrm{A}}$ symmetry is broken explicitly. The unspecified quantities $\Delta \Gamma_{\mathrm{gf}}$ and $\Delta \Gamma_{\mathrm{gh}}$ in Eq. (3) are the standard background-field gauge-fixing and ghost term, respectively. In all explicit calculations, we have restricted ourselves to Feynman gauge for convenience.
A few comments are still in order at this point: In this work, we are aiming at a study of dense strong-interaction matter. To this end, we employ the diquark field as an effective degree of freedom to analyze the properties of the ground state. Since the diquark field is not a color-neutral object, the dynamical generation of a finite expectation value of this field would break the SU(3) color symmetry and therefore gauge invariance. Of course, it is known that local gauge invariance cannot be broken [97]. Moreover, the diquarks are effective degrees of freedom which do not even need to be asymptotic states in the spectrum. In any case, in (color-)superconducting systems, the physics is governed by the formation of a gap in the spectrum of fermionic excitations at the Fermi surface and the existence of such a gap is a gauge-invariant statement. The description of the formation of this gap in the fermionic excitation spectrum in terms of a diquark condensate within a fixed gauge, which effectively breaks the gauge symmetry, is only a convenient choice to get access to the low-energy dynamics [2]. In this work, we expand the effective action in the quantity $\Delta_{a}^{*} \Delta_{a}$ (summation over $a$ is tacitly 
assumed), which is a gauge-invariant object. The gap in the fermionic spectrum is also constructed from this quantity. In practice, we employ a homogeneous background for the expansion and eventually evaluate the flow equations on a specific background configuration. This configuration is chosen to point into the 3-direction in color space for convenience, which may possibly lead to a residual dependence of our results for the gap on this choice. In future studies, our presently employed convenient approach to study the physics of dense QCD matter may be "outperformed" by directly computing the full momentum dependence of fermonic correlation functions in a vertex expansion and searching for signatures of a gap in these quantities, without relying on the use of diquark fields as auxiliary degrees of freedom. However, this is beyond the scope of the present work. We add that, in principle, similar issues are encountered in the description of mass generation in the electroweak sector of the Standard Model [98-102].

Of course, by construction, our ansatz for $\Gamma_{k}$ does not allow for a study of the transition from a color-superconducting phase at intermediate and high densities to a phase governed by spontaneous chiral symmetry breaking at low densities. Therefore, our present work focusses on the intermediate and high density regime. Note that a quantitative analysis of the regime associated with the aforementioned transition is anyhow complicated by the fact that many four-quark interaction channels have been found to be of roughly the same strength in this regime [47]. This suggests that the ground state of QCD may exhibit a very complicated structure in this transition regime. We add that, close to the nucleonic low-density regime, the dynamics may even be governed by quarkyonic matter [103].

\section{B. RG flow equations}

Let us begin our discussion of the RG flow by explaining the structure of our ansatz for the scale-dependent effective action $\Gamma_{k}$ in more detail. The initial condition for $\Gamma_{k}$ at the scale $k=\Lambda \gg \mu$ is assumed to be given by the classical QCD action (1). Therefore, the values of the four-quark coupling $\bar{\lambda}_{\text {csc }}$, the quark-diquark coupling $\bar{h}$, the bosonic wave function renormalization factor $Z_{\Delta}$, and the fourdiquark coupling $\bar{\lambda}_{\Delta}$ should be set to zero at the initial RG scale $\Lambda$. ${ }^{2}$ This choice for $Z_{\Delta}$ implies that we have $m^{2}=$ $\bar{m}^{2} / Z_{\Delta} \rightarrow \infty$ for the renormalized mass parameter of the diquarks for $k \rightarrow \Lambda$. Thus, the diquark fields are indeed not dynamical degrees of freedom at high-momentum scales. Their emergence in the low-energy regime of dense QCD matter is solely triggered by the underlying quark-gluon dynamics.

\footnotetext{
${ }^{2}$ We also refer to Sec. III A for a discussion of the initial conditions and the RG flow at high momentum scales.
}

By lowering the RG scale, starting from $k=\Lambda$, the quark-gluon vertex generates four-quark self-interactions via two-gluon exchange. With respect to this type of interaction channels, we only take into account the diquark channel as discussed above. This channel is associated with the coupling $\bar{\lambda}_{\text {csc }}$ in Eq. (3). Once generated, this four-quark interaction channel can be removed by mapping it onto a Yukawa-type quark-diquark interaction channel associated with the coupling $\bar{h}$ and a term bilinear in the diquark fields associated with the term $\sim \bar{m}^{2}$. Essentially, this corresponds to performing a Hubbard-Stratonovich transformation at a given RG scale. In the next RG step, however, the fourquark interaction $\bar{\lambda}_{\text {csc }}$ is regenerated by the quark-gluon vertex and the quark-diquark vertex. The regenerated fourquark channel can then again be removed by mapping it onto the quark-diquark interaction channel and the term bilinear in the diquark fields. Moreover, the running of the quark-diquark coupling $\bar{h}$ and the parameter $\bar{m}^{2}$ receive additional contributions from, e.g., the running of the wave function renormalization $Z_{\Delta}$ of the diquark fields. The latter is generated itself by the quark-diquark coupling $\bar{h}$. Note that, once the diquark wave function renormalization is rendered finite, the diquarks become dynamical degrees of freedom in the RG flow. It is also important to add that higher-order diquark self-interaction terms are generated via the aforementioned quark-diquark interactions. In the following, we take into account diquark self-interactions up to the four-diquark channel which is associated with the coupling $\bar{\lambda}_{\Delta}$ in Eq. (3). ${ }^{3}$ Taking another RG step, the fourquark interaction channel is then generated again and the aforementioned procedure of mapping it onto a Yukawatype quark-diquark interaction channel and a term bilinear in the diquark fields can be repeated. The repeated application of this mapping can be recast into flow equations which eventually allow us to follow the RG flow from the perturbative high-momentum regime governed by quark-gluon dynamics down to the low-energy regime governed by the formation of bound states of quarks. Within the functional RG framework, this procedure can be implemented with the aid of the so-called dynamical hadronization technique [68-75], as already indicated in the previous subsection.

Employing this technique, see Appendix B for details, we find the following coupled set of flow equations for the dimensionless renormalized curvature of the effective potential $\epsilon_{\mu}=\left(\bar{m}^{2}-4 Z_{\Delta} \mu^{2}\right) Z_{\Delta}^{-1} k^{-2}$, the renormalized four-diquark coupling $\lambda_{\Delta}=\bar{\lambda}_{\Delta} Z_{\Delta}^{-2}$, and the renormalized quark-diquark coupling $h=\bar{h} Z_{\Delta}^{-\frac{1}{2}}$ :

\footnotetext{
${ }^{3}$ Such diquark self-interaction channels can be related to higher-order quark self-interaction channels with a nontrivial momentum structure. For example, the four-diquark channel can be related to an eight-quark interaction channel.
} 


$$
\begin{aligned}
\partial_{t} \epsilon_{\mu}= & \left(\eta_{\Delta}-2\right) \epsilon_{\mu}-8 h^{2} b_{(2,0)}(\tilde{\mu}, 0) \\
& +\frac{2}{h^{2}} \epsilon_{\mu}\left(1+\epsilon_{\mu}\right) g^{4} b_{(0,4)}^{(A)}\left(\tilde{\mu}, 0, \eta_{A}\right), \\
\partial_{t} \lambda_{\Delta}= & 2 \eta_{\Delta} \lambda_{\Delta}+4 h^{4} b_{(4,0)}(\tilde{\mu}, 0) \\
& +4 \frac{\lambda_{\Delta}}{h^{2}}\left(1+\epsilon_{\mu}\right) g^{4} b_{(0,4)}^{(A)}\left(\tilde{\mu}, 0, \eta_{A}\right), \\
\partial_{t} h^{2}= & \eta_{\Delta} h^{2}+\frac{16}{3} g^{2} h^{2} b_{(1,2)}^{(A)}\left(\tilde{\mu}, 0, \eta_{A}\right) \\
& +2\left(1+2 \epsilon_{\mu}\right) g^{4} b_{(0,4)}^{(A)}\left(\tilde{\mu}, 0, \eta_{A}\right),
\end{aligned}
$$

where $\tilde{\mu}=\mu / k$ is the dimensionless chemical potential and $g^{2}=\bar{g}^{2} Z_{A}^{-1}$ is the renormalized strong coupling. Finally, the scale-dependence of the anomalous dimension of the diquark fields is governed by

$$
\eta_{\Delta}=-\partial_{t} \ln Z_{\Delta}=8 h^{2} d_{(2,0)}(\tilde{\mu}, 0) .
$$

Recall that the set of couplings associated with these equations span our ansatz (3) for the scale-dependent effective action $\Gamma_{k}$. The functions $b_{(i, j)}$ and $d_{(i, j)}$ are socalled threshold functions which correspond to one-particle irreducible (1PI) Feynman diagrams with $i$ external bosonic and $j$ external fermionic lines, respectively. In some cases, an additional superscript $(A)$ appears which indicates that the associated diagram contains at least one gluon line. Since we restrict ourselves to the zero-temperature limit in this work, these functions only depend on the dimensionless chemical potential $\tilde{\mu}$ in the absence of a diquark gap. In any case, the regularization scheme dependence is also encoded in these functions. In this respect, we note that we employ a scheme which allows us to integrate out fermionic fluctuations around the Fermi surface [78], see Appendix A for its definition and brief discussion of all threshold functions entering our present study. It should be emphasized that also the anomalous dimensions $\eta_{\Delta}$ and $\eta_{A}=$ $-\partial_{t} \ln Z_{A}$ depend on the dimensionless chemical potential $\tilde{\mu}$. The running of the strong coupling $g$ and its relation to the wave function renormalization $Z_{A}$ of the gauge fields is discussed below.

By comparing the set of flow equations (4)-(7) with our ansatz (3) for the effective action, it becomes apparent that there is no flow equation for the four-quark coupling $\bar{\lambda}_{\mathrm{csc}}$. With the aid of the aforementioned dynamical hadronization technique [68-74], the contributions to this coupling are continuously transformed into contributions to the flow of the quark-diquark coupling $h$ and the curvature $\epsilon_{\mu}$, such that $\partial_{t} \bar{\lambda}_{\mathrm{csc}}=0$ for any value of $k$. The contributions to the flow of the four-quark coupling $\bar{\lambda}_{\text {csc }}$ therefore appear in the flow equations for the quark-diquark coupling $h$ and the curvature $\epsilon_{\mu}$. In particular, these contributions are associated with the terms $\sim g^{4}$ in the flow equations (4) and (6) which originally stem from two-gluon exchange box diagrams appearing in the $\mathrm{RG}$ flow of four-quark couplings. ${ }^{4}$

The set of flow equations (4)-(7) describes the dynamics at high-momentum scales where the curvature $\epsilon_{\mu}$ of the effective potential is positive. In fact, as discussed above, we shall choose initial conditions such that $\epsilon_{\mu} \gg 1$, $\lambda_{\Delta} \rightarrow 0$, and $h^{2} \rightarrow 0$ for $k \rightarrow \Lambda$. Quark self-interactions, which are mapped onto diquark self-interactions and quarkdiquark interactions in our present setting, are initially only generated by two-gluon exchange $\sim g^{4}$. Following the RG flow to smaller scales $k$, we find that the curvature $\epsilon_{\mu}$ decreases and eventually becomes zero at a finite scale $k_{\mathrm{SB}}$, see also our discussion in Sec. III below. At the scale $k_{\mathrm{SB}}$, spontaneous $\mathrm{U}(1)_{\mathrm{V}}$ symmetry breaking sets in.

Below the symmetry breaking scale $k_{\mathrm{SB}}$, the curvature $\epsilon_{\mu}$ of the effective potential becomes negative and a colorsuperconducting ground state is formed associated with the formation of a gap in the fermionic excitation spectrum. Note that the antisymmetric flavor structure of this colorsuperconducting ground state corresponds to a singlet representation of the global chiral group. This implies that the formation of such a ground state does not violate the chiral symmetry. In any case, for $k \leq k_{\mathrm{SB}}$, it is convenient to switch from the set of flow equations (4)-(6) to a set in which the flow equation for the curvature $\epsilon_{\mu}$ is replaced with a flow equation for the minimum $\left|\Delta_{0}\right|^{2}=\sum_{a}\left|\Delta_{0, a}\right|^{2}$. Recall that we expand the effective action in the quantity $\Delta_{a}^{*} \Delta_{a}$ (summation over $a$ is assumed). For convenience, we shall choose $\Delta_{0, a}=\Delta_{0} \delta_{a, 3}\left(\Delta_{0} \in \mathbb{R}\right)$ and use $\kappa=$ $Z_{\Delta}\left|\Delta_{0}\right|^{2} k^{-2}$ to parametrize the flow of the position of the minimum of the effective action. The resulting set of flow equations for scales $k<k_{\mathrm{SB}}$ then reads

$$
\begin{aligned}
\partial_{t} \kappa= & -\left(\eta_{\Delta}+2\right) \kappa+\frac{4 h^{2}}{\lambda_{\Delta}} b_{(2,0)}\left(\tilde{\mu}, h^{2} \kappa\right), \\
\partial_{t} \lambda_{\Delta}= & 2 \eta_{\Delta} \lambda_{\Delta}+4 h^{4} b_{(4,0)}\left(\tilde{\mu}, h^{2} \kappa\right) \\
& +4 \frac{\lambda_{\Delta}}{h^{2}} g^{4} b_{(0,4)}^{(A)}\left(\tilde{\mu}, h^{2} \kappa, \eta_{A}\right), \\
\partial_{t} h^{2}= & \eta_{\Delta} h^{2}+\frac{16}{3} g^{2} h^{2} b_{(1,2)}^{(A)}\left(\tilde{\mu}, h^{2} \kappa, \eta_{A}\right) \\
& +2 g^{4} b_{(0,4)}^{(A)}\left(\tilde{\mu}, h^{2} \kappa, \eta_{A}\right),
\end{aligned}
$$

\footnotetext{
${ }^{4}$ It is indeed possible to recover the flow equation for the fourquark coupling $\bar{\lambda}_{\text {csc }}$ at scales above the symmetry breaking scale $k_{\mathrm{SB}}$. To be more specific, we have $\lambda_{\mathrm{csc}}=h^{2} /\left(2 \epsilon_{\mu}\right)$ for the dimensionless four-quark coupling which relates the dimensionless four-quark coupling to the quark-diquark coupling $h$ and the curvature $\epsilon_{\mu}$. From this flow equation, we deduce that the RG flow at sufficiently large scales $k$ is governed by the two fixed points of $\lambda_{\mathrm{csc}}$, provided the strong coupling $g^{2}$ is sufficiently small, see Sec. III C. These fixed points can be translated into fixed points for the quark-diquark coupling $h$ and the curvature $\epsilon_{\mu}$, see Ref. [62] for a general discussion of this aspect.
} 
and

$$
\eta_{\Delta}=8 h^{2} d_{(2,0)}\left(\tilde{\mu}, h^{2} \kappa\right) .
$$

In addition to the dimensionless chemical potential $\tilde{\mu}$, the anomalous dimensions $\eta_{\Delta}$ and $\eta_{A}$ now also depend on the so-called (diquark) gap $\left|\Delta_{\text {gap }}\right|$ which appears in the propagator of the quarks. In our conventions, we have $\left|\Delta_{\text {gap }}\right|=$ $h \sqrt{\kappa} k$. Thus, the gap $\left|\Delta_{\text {gap }}\right|$ in the quark propagator is directly related to the minimum $\left|\Delta_{0}\right|^{2}$.

The gauge sector enters our flow equations (4)-(11) only via the running of the strong coupling $g$ which is governed by the following equation:

$$
\partial_{t} g^{2}=\eta_{A} g^{2}
$$

Here, $\eta_{A}$ can be decomposed into a pure gluonic contribution $\eta_{\text {glue }}$ and a term $\eta_{q}$ which contains the quark contributions [63-65]:

$$
\eta_{A}=-\partial_{t} \ln Z_{A}=\eta_{\text {glue }}+\eta_{q}
$$

For the purely gluonic contribution $\eta_{\text {glue }}$, we employ the results from previous functional RG studies $[64,65,86]$. There, $\eta_{\text {glue }}$ has been computed nonperturbatively within the background field formalism which also underlies our present work. The quark contribution $\eta_{q}$ depends on the dimensionless chemical potential $\tilde{\mu}$ and the diquark gap:

$$
\eta_{q}=\frac{1}{4} g^{2} d_{(2,0)}^{(A)}\left(\tilde{\mu}, h^{2} \kappa\right)
$$

In the limit $\tilde{\mu} \rightarrow 0$ and $h^{2} \kappa \rightarrow 0$, we have $d_{(2,0)}^{(A)}(0,0)=$ $2 /\left(3 \pi^{2}\right)$ and therefore $\eta_{q}=g^{2} /\left(6 \pi^{2}\right)$. This is nothing but the standard one-loop contribution of the quark fields to the running of the strong coupling $g$. We add that, in general, the running of the gauge coupling also receives corrections from quark self-interactions, such as four-quark interactions, see, e.g., Refs. [104,105]. However, within the fRG framework, it follows from an analysis of (modified) Ward-Takahashi identities that such back-reactions of the matter sector on the gauge sector are negligible, provided that the flow of the four-quark couplings is governed by the presence of fixed points [63-65]. At least above the symmetry breaking scale $k_{\mathrm{SB}}$, this is indeed the case in our present study (see also Sec. III A) which justifies that we do not take such contributions to the running of the gauge sector into account, see also Ref. [47]. For $k<k_{\mathrm{SB}}$, we shall neglect such contributions. Note that, in this regime, the situation is particularly involved anyhow because of the presence of a finite quark gap, as we shall discuss next and also in Sec. III below.

In our flow equations (4)-(14) we drop fluctuations of the diquark fields. Such fluctuation effects are associated with 1PI diagrams coming with at least one internal diquark line. Compared to the contributions that we take into account in our analysis, such contributions are subleading in an $N_{\mathrm{c}}$-counting. Moreover, in the symmetric high-energy regime (i.e., for $k>k_{\mathrm{SB}}$ ), the fluctuation effects of the diquark fields are parametrically suppressed because of the large diquark mass parameter. In Sec. III, we shall see that this parameter is indeed large and only becomes small close to the symmetry breaking scale $k_{\mathrm{SB}}$. Such a suppression of fluctuation effects has already been observed and discussed in early fRG studies of chiral models in the zero-density limit [106-108].

In the regime $k<k_{\mathrm{SB}}$, which is governed by spontaneous symmetry breaking, it can no longer be argued that fluctuation effects are subleading. Whereas fluctuation effects are associated with, e.g., pion dynamics at low densities, a rigorous inclusion of fluctuations of the diquark fields at high densities requires to deal with an AndersonHiggs-type mechanism [98-102] associated with the symmetry-breaking pattern $\mathrm{SU}(3) \rightarrow \mathrm{SU}(2)$ in color space (as the diquark fields carry a net color charge). As a consequence, only three of the eight gluons are massless. The remaining five gluons are effectively rendered massive by, loosely speaking, "eating up" Goldstone modes which appear in the diquark spectrum in the symmetry-broken regime, see, e.g., Ref. [6] for a review. In our present study, which mainly aims at setting the methodological stage for future more quantitative studies of dense QCD matter, we do not include this Anderson-Higgs-type mechanism but rather drop diquark fluctuations as mentioned above. A more quantitative study taking this Anderson-Higgs-type mechanism into account is deferred to future work. The general methodological groundwork for studies of this type of mechanism within the fRG framework has already been laid in studies of Abelian Higgs models [109,110] and (non-Abelian) gauged chiral Higgs-Yukawa models [111]. In any case, we shall at least estimate the effect of the appearance of the associated gap for the gluons on our present results in Sec. III below.

\section{RG FLOW OF DENSE QCD MATTER}

\section{A. Scale fixing}

Let us now discuss our results for the RG flow of dense QCD matter, in particular those for the chirally symmetric (scalar) diquark condensate. To this end, we first need to specify the initial conditions of our RG flow equations at the UV scale $k=\Lambda=10 \mathrm{GeV}$. This value of the initial scale ensures that we have $\Lambda \gg \mu$ for all values of the quark chemical potential considered in the present work. For the dimensionless renormalized curvature $\epsilon_{\mu}$ of the effective potential, we choose $\epsilon_{\mu}=10^{6}$. Thus, the diquark fields do not represent dynamical degrees of freedom at the UV scale $\Lambda$. We add that the limit $\epsilon_{\mu} \rightarrow \infty$ corresponds to the limit of a vanishing diquark wave function renormalization, $Z_{\Delta} \rightarrow 0$. 

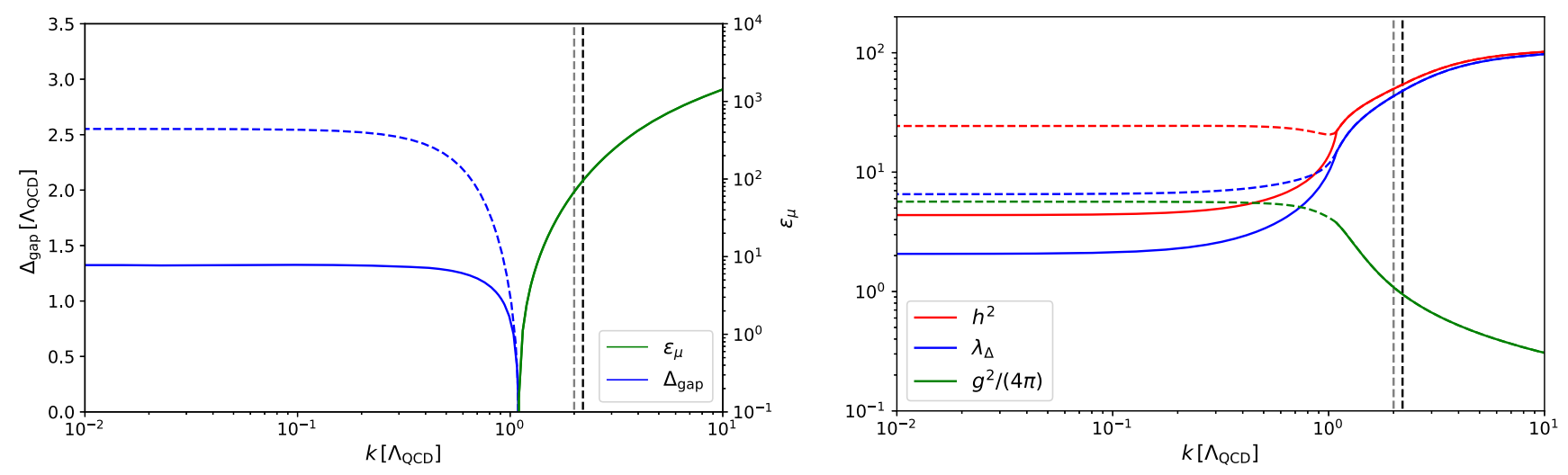

FIG. 1. RG flow of the renormalized dimensionless curvature $\epsilon_{\mu}$ for $k \geq k_{\mathrm{SB}}$ (left panel), the diquark gap $\Delta_{\text {gap }}$ for $k \leq k_{\mathrm{SB}}$ (left panel), the (squared) renormalized quark-diquark coupling $h^{2}$ (right panel), the renormalized four-diquark coupling $\lambda_{\Delta}$ (right panel), and the renormalized strong coupling $\alpha=g^{2} /(4 \pi)$ (right panel) for $\mu / \Lambda_{\mathrm{QCD}}=2$, where $k_{\mathrm{SB}} / \Lambda_{\mathrm{QCD}} \approx 1.09$. In both panels, the gray (vertical) dashed line is associated with the scale $k=\mu$. The black (vertical) dashed line in these panels is associated with the scale $k=k_{\mathrm{m}}$. Here, $k_{\mathrm{m}}$ is an estimate for the scale at which the gluon screening masses exceed the scale $k$. For $k<k_{\mathrm{SB}}$, the results for the diquark gap $\Delta_{\text {gap }}$ (left panel) and the couplings in the right panel are given as solid and dashed lines. The dashed lines represent the running of these quantities for the case in which the gluons remain ungapped and do not acquire a mass according to the Anderson-Higgs mechanism below the symmetry breaking scale $k_{\mathrm{SB}}$. The solid lines show the results for the case in which the gluons have been fully decoupled from the matter sector for $k \leq k_{\mathrm{SB}}$.

For the quark-diquark coupling $h$, we choose $h=0.1$ at $k=\Lambda$. The initial value of the four-diquark coupling $\lambda_{\Delta}$ is set to zero. This choice for the couplings at the scale $\Lambda$ ensures that we indeed initialize the flow "in the vicinity of" the QCD action in the UV limit. ${ }^{5}$ Note that we have checked that our results in the IR limit (in particular those for the diquark gap) depend only very weakly on the specific choice for the initial values of the couplings, provided that we ensure $\Lambda \gg k_{\mathrm{SB}}$. This independence can be traced back to the appearance of pseudo fixed points in the RG flow of the gluon-induced interaction channels, ${ }^{6}$ see Refs. $[69,71-74]$ for a detailed discussion of this aspect in the vacuum limit of QCD. The appearance of a pseudo fixed-point behavior at finite chemical potential together with a loss of memory of the details of the initial conditions may already be anticipated from an analysis of the fixed-point structure of gluon-induced four-quark interaction channels, see Ref. [47] for details. Indeed, the quark-diquark coupling and the curvature of the effective potential can be directly related to the four-quark coupling $\lambda_{\mathrm{csc}}=h^{2} /\left(2 \epsilon_{\mu}\right)$. Therefore, fixed points of the four-quark coupling $\lambda_{\text {csc }}$ leave their imprint in the RG flows of the quark-diquark coupling $h$ and the curvature $\epsilon_{\mu}$. For example, our choice $\epsilon_{\mu} \gg h^{2}$ at the UV scale implies that

\footnotetext{
${ }^{5} \mathrm{We}$ add that a finite value of the quark-diquark coupling $h$ explicitly breaks the $\mathrm{U}(1)_{\mathrm{A}}$ symmetry. As discussed in, e.g., Ref. [47], this is required to render the four-quark coupling $\lambda_{\text {csc }}$ associated with the diquark channel $\sim\left(\bar{\psi}_{b} \tau_{2} \epsilon_{a b c} \gamma_{5} \mathcal{C} \bar{\psi}_{c}^{T}\right)$ $\left(\psi_{d}^{T} \mathcal{C} \gamma_{5} \tau_{2} \epsilon_{a d e} \psi_{e}\right)$ to be most dominant at high densities [47].

${ }^{6}$ In the present study, we only encounter pseudo fixed points since a dimensionful scale enters the RG flow via the quark chemical potential.
}

we initialize the RG flow (very) close to the Gaußian fixed point of the four-quark coupling $\lambda_{\text {csc }}$.

From this discussion it follows that the initial value of the strong coupling is the only input parameter in our calculations. It sets the scale for all dimensionful quantities. In our present study with two massless quark flavors, we choose $\alpha=g^{2} /(4 \pi)=0.179 \pm 0.004$ at the UV scale $\Lambda=10 \mathrm{GeV}$ which corresponds to the experimental value $\alpha=0.330 \pm 0.014$ at the $\tau$-mass scale [112]. ${ }^{7}$ For $\Lambda_{\mathrm{QCD}}-$ defined as the inflection point of the strong coupling - we then obtain $\Lambda_{\mathrm{QCD}} \approx 209 \mathrm{MeV}$ in the vacuum limit. From here on, we shall measure all dimensionful quantities in units of $\Lambda_{\mathrm{QCD}}$. For example, we have $\Lambda / \Lambda_{\mathrm{QCD}} \approx 47.8$.

\section{B. From quark-gluon dynamics to color superconductivity}

In Fig. 1, we show the RG flow of the renormalized dimensionless curvature $\epsilon_{\mu}$ for $k \geq k_{\mathrm{SB}}$ (left panel), the diquark gap $\Delta_{\text {gap }}$ for $k \leq k_{\mathrm{SB}}$ (left panel), the (squared) quark-diquark coupling $h^{2}$ (right panel), the four-diquark coupling $\lambda_{\Delta}$ (right panel), and the strong coupling $\alpha=$ $g^{2} /(4 \pi)$ (right panel) over a wide range of scales for $\mu / \Lambda_{\mathrm{QCD}}=2$. In this case, we have $k_{\mathrm{SB}} / \Lambda_{\mathrm{QCD}} \approx 1.09$. The gray (vertical) dashed lines in the two panels represent the point in the RG flow where $k=\mu$. The black (vertical) dashed lines are associated with the scale $k=k_{\mathrm{m}}$. The latter is an estimate for the scale at which the screening masses of

\footnotetext{
${ }^{7}$ Note that the running of the strong coupling entering our calculation is compatible with the standard $\overline{\mathrm{MS}}$ running over a wide range of scales $[64,65,86]$.
} 
the gluons exceed the scale $k{ }^{8}$ Here, we estimate this scale from the relation $m_{\mathrm{g}}=g\left(k_{\mathrm{m}}\right) \mu / \pi=k_{\mathrm{m}}$, where $m_{\mathrm{g}}$ represents an estimate for the gluon screening masses in the symmetric high-energy regime $\left(k>k_{\mathrm{SB}}\right)$, see, e.g., Refs. [113,114]. Note that these masses are schemedependent quantities. A detailed analysis of this aspect will be given elsewhere [115]. In any case, for small chemical potentials (e.g., $\mu / \Lambda_{\mathrm{QCD}}=2$ as shown in Fig. 1), we observe a hierarchy of scales: $k_{\mathrm{SB}}<\mu<k_{\mathrm{m}}$.

For $k>k_{\mathrm{m}}$, gluon screening effects are parametrically suppressed since $m_{\mathrm{g}} / k<1$. Note that effects associated with the quark chemical potential appearing in the quark propagator are even more suppressed, $\mu / k<m_{\mathrm{g}} / k<1$. In this high-energy regime, we therefore do not expect that our results suffer significantly from the fact that we have neglected the gluon screening masses in our calculations. For these scales, the RG flow of the couplings is mainly driven by gluon exchange diagrams. Following the RG flow toward smaller scales, the strong coupling increases (see right panel of Fig. 1) and gauge fluctuations tend to drive the system toward a ground state associated with a (spontaneously) broken $\mathrm{U}(1)_{\mathrm{V}}$ symmetry. However, it should be noted that strong gauge fluctuations are in principle not required to trigger the formation of a (color-)superconducting ground state because of the presence of a Cooper instability in the system, ${ }^{9}$ see Refs. [46,76] for a general fixed-point analysis of this aspect and Ref. [48] for an early mean-field study in QCD. The gauge fluctuations rather act as a "catalyzer" for the formation of a (color-)superconducting ground state. Loosely speaking, strong gauge fluctuations tend to increase the symmetry breaking scale $k_{\mathrm{SB}}$ and therefore also the diquark gap $\Delta_{\text {gap }} \sim k_{\mathrm{SB}}$. In other words, without strong gauge fluctuations, the diquark gap would be (significantly) smaller.

From this line of arguments it is already clear that gluon screening effects become relevant at some point in the RG flow toward the infrared regime. To be more specific, we expect that the presence of gluon screening masses affects the dynamics for $k_{\mathrm{SB}}<k<k_{\mathrm{m}}$. In this regime, contributions to the RG flow with at least one internal gluon line start to become parametrically suppressed since we have $m_{\mathrm{g}} / k>1$. This reduces the aforementioned "catalyzing effect" of the gluons and presumably leads to a shift of the symmetry breaking scale $k_{\mathrm{SB}}$ and the gap $\Delta_{\text {gap }}$ to smaller values compared to the ones obtained in our present study. A detailed analysis of this aspect will be given elsewhere. In any case, we expect that their inclusion will not significantly alter the value of the symmetry breaking scale $k_{\mathrm{SB}}$ or the dynamics

\footnotetext{
${ }^{8}$ For simplicity, we do not distinguish between the electric and magnetic masses.

${ }^{9}$ This is different for chiral symmetry breaking which requires the gauge coupling to become sufficiently large, see, e.g., Refs. [47,62-65] for a detailed discussion.
}

for $k_{\mathrm{SB}}<k<k_{\mathrm{m}}$, at least for sufficiently small values of the chemical potential. For example, for $\mu / \Lambda_{\mathrm{QCD}}=2$, we have $k_{\mathrm{SB}} / \Lambda_{\mathrm{QCD}} \approx 1.1$ and $k_{\mathrm{m}} / \Lambda_{\mathrm{QCD}} \approx 2.2$. Consequently, gluon screening effects are expected to be relevant only in a comparatively small regime above the symmetry breaking scale.

For increasing chemical potential, we find that the symmetry breaking scale $k_{\mathrm{SB}}$ increases but only mildly, see also Fig. 2 and our discussion in Sec. III C below. In any case, a change in the hierarchy of scales sets in for increasing $\mu$, where we eventually have $k_{\mathrm{SB}}<k_{\mathrm{m}}<\mu$. The quark dynamics is now strongly affected by the presence of the chemical potential over a wide range of scales. For $\mu / \Lambda_{\mathrm{QCD}}=10$, for example, we have $k_{\mathrm{SB}} / \Lambda_{\mathrm{QCD}} \approx 1.6$. The gluon screening masses are smaller than the quark chemical potential over a wide range of scales within the regime $k_{\mathrm{SB}}<k_{\mathrm{m}}<\mu$. Nevertheless, these screening masses increase roughly linearly when $\mu$ is increased. For a given scale $k$, this suggests a stronger (parametric) suppression of gluonic contributions to the RG flow at large chemical potential than at small chemical potential. In other words, gluon screening effects may have a stronger impact on the RG flow over a wider range of scales when the chemical potential is increased. Correspondingly, the aforementioned "catalyzing effect" of the gauge degrees of freedom is expected to be reduced. Therefore, it is reasonable to expect that our estimates for the symmetry breaking scale $k_{\mathrm{SB}}$ and the diquark gap become less reliable for large chemical potentials. In fact, after the conventional BCS-type increase of $k_{\mathrm{SB}}$ for small chemical potentials, this suggests that gluon screening effects may potentially even lead to a decrease of $k_{\mathrm{SB}}$ over some range of quark chemical potentials. For chemical potentials beyond those considered in this work, however, it is known that the diquark gap increases again as a function of the chemical potential [43], see also Refs. [44,45,52,54,56,116] for a discussion of the relevance of gluon screening effects.

Let us now turn to the regime $k<k_{\mathrm{SB}}$ associated with spontaneous $\mathrm{U}(1)_{\mathrm{V}}$ breaking. In this regime, the situation is even more involved as it requires to deal with an AndersonHiggs-type mechanism [98-102] associated with the breaking of the $\mathrm{SU}(3)$ symmetry in color space down to a $\mathrm{SU}(2)$ symmetry. This eventually leads to the generation of "gaps" (screening masses) for five of the eight gluons. A rigorous treatment of this mechanism is beyond the scope of the present work. We only consider two approximations in the low-energy regime $k<k_{\mathrm{SB}}$ to already gain some understanding of the effect of gluon screening in the longrange limit.

In the first approximation, we simply leave the gluons ungapped for $k<k_{\mathrm{SB}}$. The corresponding results for the RG flow of the diquark gap $\Delta_{\text {gap }}$ and the various couplings are depicted by the dashed lines in Fig. 1 (and also in Fig. 2). In the second approximation associated with the solid lines for $k<k_{\mathrm{SB}}$ in Fig. 1 (and also in Fig. 2), we 

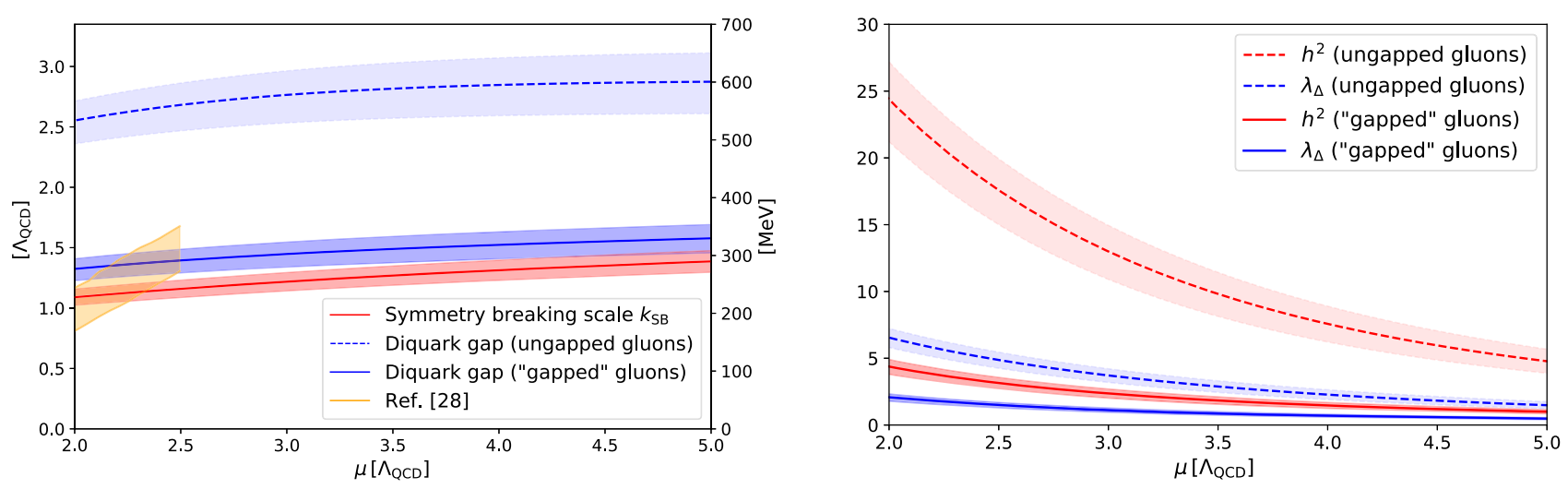

FIG. 2. Left panel: diquark gap $\Delta_{\text {gap }}$ and the symmetry-breaking scale $k_{\mathrm{SB}}$ as a function of the quark chemical potential $\mu$. The shaded bands (apart from the orange band) represent the uncertainty resulting from a variation of the strong coupling at the initial RG scale. The blue dashed line together with the light blue band (ungapped gluons) represent the gap for the case where the gluons remain ungapped below the symmetry breaking scale $k_{\mathrm{SB}}$. The solid blue line together with the dark blue band ("gapped" gluons) show the gap for the case where the gluons have been decoupled from the matter sector for $k \leq k_{\mathrm{SB}}$, see main text for details. The orange band depicts results for the diquark gap from a previous fRG study [28]. Note that, in Ref. [28], the shown range of chemical potentials is associated with densities $n / n_{0} \approx 6 \ldots 12$ (where $n_{0}$ is the nuclear saturation density). The results from Ref. [28] are in remarkable agreement with those from early studies of the diquark gap for $n / n_{0} \lesssim 5$ [48]. For $n / n_{0} \approx 5$, for example, $\Delta_{\text {gap }} \approx 70 \ldots 160 \mathrm{MeV}$ was reported in Ref. [48] and $\Delta_{\text {gap }} \approx 140 \ldots 230 \mathrm{MeV}$ was found in Ref. [28]. Right panel: the (squared) renormalized quark-diquark coupling $h^{2}$ and the renormalized four-diquark coupling $\lambda_{\Delta}$ as a function of the quark chemical potential $\mu$. Dashed and solid lines are again associated with ungapped and "gapped" gluons in the low-energy regime, respectively. The shaded bands represent the uncertainty resulting from a variation of the strong coupling at the initial RG scale.

decouple the gluon contributions from the RG flow of the matter sector, which may be viewed as adding an "infinite gap" to all gluons. In practice, we have implemented this decoupling by setting the gauge coupling to zero for $k<k_{\mathrm{SB}}$. Comparing the corresponding results with the ones for the ungapped gluons, we observe that the diquark gap is reduced by roughly a factor of two. As already discussed above, ungapped/unscreened gluons indeed act as a "catalyzer" for the formation of a (color-) superconducting ground state. This remains also true when the chemical potential is increased, see Fig. 2. For the renormalized quark-diquark coupling $h$ and the renormalized four-diquark coupling $\lambda_{\Delta}$, we observe a similar behavior. The couplings receive a significant boost in the approximation with ungapped gluons, see the right panels of Figs. 1 and 2.

\section{Diquark gap}

Our results for the diquark gap $\Delta_{\text {gap }}$, the symmetry breaking scale $k_{\mathrm{SB}}$, the quark-diquark coupling $h$, and the four-diquark coupling $\lambda_{\Delta}$ in the limit $k \rightarrow 0$ as a function of the quark chemical potential are summarized in Fig. 2. The (shaded) bands in Fig. 2 result from a variation of the strong coupling at the initial RG scale, see Sec. III A. Note that the variation of our results arising from a variation of the regularization scheme (associated with regulator functions) is negligible compared to the one obtained from the aforementioned variation of the initial value of the strong coupling. We refer the reader to Appendix A for the definition of the regulator functions employed in the present work and a corresponding discussion of the scheme dependence.

The observed dependence of the symmetry breaking scale $k_{\mathrm{SB}}$ on the chemical potential appears consistent with the standard BCS-type scaling behavior. This is true for the case with ungapped gluons in the low-energy regime and for the case with decoupled gluon contributions as associated with (infinitely) "gapped" gluons. However, the diquark gap in the case with ungapped gluons is found to be significantly greater than the one obtained in our calculations with "gapped" gluons.

Let us now analyze the scaling behavior of the symmetry breaking scale and the diquark gap in more detail. To this end, it is convenient to reconstruct the RG flow of the fourquark coupling $\lambda_{\mathrm{csc}}=h^{2} /\left(2 \epsilon_{\mu}\right)$ from the RG flows of the quark-diquark coupling $h$ and the curvature $\epsilon_{\mu}$ of the effective potential. Employing Eqs. (4) and (6), we then find

$\partial_{t} \lambda_{\mathrm{csc}}=2 \lambda_{\mathrm{csc}}+16 \lambda_{\mathrm{csc}}^{2} b_{(2,0)}+\frac{16}{3} \lambda_{\mathrm{csc}} g^{2} b_{(1,2)}^{(A)}+g^{4} b_{(0,4)}^{(A)}$.

Setting $\lambda_{\mathrm{csc}}=0$ (corresponding to $\epsilon_{\mu} \gg h^{2}$ ) at the initial RG scale $k=\Lambda \gg \mu$, the RG flow of $\lambda_{\text {csc }}$ is then dominated by the contributions $\sim g^{4}$ associated with two-gluon exchange diagrams. All the other contributions to the flow 
of this coupling are initially subleading. Thus, we are left with

$$
\partial_{t} \lambda_{\mathrm{csc}}=2 \lambda_{\mathrm{csc}}+g^{4} b_{(0,4)}^{(A)},
$$

where $b_{(0,4)}^{(A)}<0$ for $\mu / k \rightarrow 0$, see Appendix A.

Let us now define a scale $\bar{k}$ such that the dependence of the two-gluon exchange diagrams on the chemical potential is negligible for $k>\vec{k}$. In this regime, the flow equation (16) can be solved analytically. Integrating Eq. (16) from $k=\Lambda$ down to $k=\bar{k}$, we find

$$
\lambda_{\mathrm{csc}}(\bar{k})=-\frac{1}{2} b_{(0,4)}^{(A)} g^{4}(\bar{k})+\mathcal{O}\left(g^{6}\right)
$$

Here, we dropped terms which are subleading for $\Lambda \gg \bar{k}$.

We shall now also assume that $\bar{k}$ can be chosen such that, at this scale, the gluon-induced four-quark self-interactions $\sim \lambda_{\text {csc }}$ have become strong enough to "dominate" their own RG flow. For sufficiently small values of the chemical potential $\mu$, it may indeed be possible to choose $\bar{k}$ such that the approximations underlying the derivations of Eqs. (16) and (17) are still at least reasonable. For $k<\bar{k}$, the flow equation (15) of the four-quark coupling $\lambda_{\mathrm{csc}}$ then reduces to

$$
\partial_{t} \lambda_{\mathrm{csc}}=2 \lambda_{\mathrm{csc}}+16 \lambda_{\mathrm{csc}}^{2} b_{(2,0)}
$$

where $b_{(2,0)}<0$ for $\mu / k \rightarrow 0$, see Appendix A.

The initial condition for the flow equation (18) at $k=\bar{k}$ is given by Eq. (17). Note that $b_{(2,0)}$ is associated with a purely fermionic one-loop diagram with only two internal fermion lines and four external fermion lines.

From the flow equation (18) we can now obtain an estimate for the symmetry breaking scale $k_{\mathrm{SB}}$. Indeed, this scale is defined as the scale at which the curvature $\epsilon_{\mu}$ of the effective potential becomes zero, i.e., the four-quark coupling $\lambda_{\mathrm{csc}}=h^{2} /\left(2 \epsilon_{\mu}\right)$ diverges at this scale. Thus, we have $1 / \lambda_{\text {csc }}\left(k_{\mathrm{SB}}\right)=0$. Next, we note that, for $k<\bar{k}$, the flow eventually enters a regime where $\mu / k>1$. In this regime, the loop diagram $\sim \lambda_{\text {csc }}^{2}$ scales as $b_{(2,0)} \sim$ $-c_{\psi}\left(\mu^{2} / k^{2}\right)$ with $c_{\psi}>0$ being a dimensionless schemedependent constant. ${ }^{10}$ With this at hand, we can solve Eq. (18) for the symmetry breaking scale and find $k_{\mathrm{SB}} \sim \bar{k} \exp \left(-c / \mu^{2}\right)$, with $c=\bar{k}^{2} /\left(16 c_{\psi} \lambda_{\mathrm{csc}}(\bar{k})\right)>0$ being a dimensionless constant. Plugging now Eq. (17) into this expression for $k_{\mathrm{SB}}$, we finally arrive at the following result for the symmetry breaking scale:

\footnotetext{
${ }^{10}$ Note that the general dependence of this four-quark interaction on $\mu$ is scheme-independent, at least for $\mu / k \gg 1$, see also Ref. [76] for a discussion.
}

$$
k_{\mathrm{SB}} \sim \exp \left(-\frac{\bar{c}}{g^{4} \mu^{2}}\right)
$$

where $\bar{c}=-\bar{k}^{2} /\left(8 c_{\psi} b_{(0,4)}^{(A)}\right)$ is a positive constant and the strong coupling is assumed to be evaluated at the scale $\bar{k} .{ }^{11}$ Since the symmetry breaking scale $k_{\mathrm{SB}}$ sets the scale for low-energy observables, such as the diquark gap, we conclude that $\Delta_{\text {gap }} \sim k_{\mathrm{SB}}$. This assumption is indeed confirmed by our numerical results, see Fig. 2.

We emphasize that our result for the dependence of the symmetry breaking scale $k_{\mathrm{SB}}$ on the strong coupling differs from the one reported in, e.g., Refs. [50,117,118], see also Ref. [43]. In these seminal studies, it was found that $\Delta_{\text {gap }} \sim k_{\mathrm{SB}} \sim \exp \left(-\bar{c}^{\prime} /\left(g^{2} \mu^{2}\right)\right)$, where $\bar{c}^{\prime}$ is a positive constant. This $g^{2}$-dependence is a consequence of the assumption $\lambda_{\text {csc }} \sim g^{2}$. Basically, the latter can be traced back to a tree-level consideration of four-quark interactions as triggered by a one-gluon exchange. In our present work, we have taken into account loop contributions to $\lambda_{\mathrm{csc}}$ which then alter the dependence of $k_{\mathrm{SB}}$ on the strong coupling as given in Eq. (19). Starting from small chemical potentials, this change in the dependence of $k_{\mathrm{SB}}$ on the strong coupling potentially induces a more rapid increase of the diquark gap $\Delta_{\text {gap }}$ when the chemical potential is increased. In any case, the scaling behavior (19) is only valid for sufficiently small values of the quark chemical potential, as discussed above. For very large chemical potentials, the diquark gap is eventually expected to increase mildly according to $\Delta_{\text {gap }} \sim$ $\mu \exp \left(-\bar{c}^{\prime \prime} / g\right.$ ) (where $\bar{c}^{\prime \prime}>0$ is a constant) [43], such that $\Delta_{\text {gap }} / \mu$ still decreases, see Ref. [6] for a detailed discussion of the diquark gap at very high densities.

We now turn to a more quantitative comparison of our present results with already existing results for the diquark gap. Of course, a direct comparison is difficult as it in principle requires to consider the diquark gap as a function of the density. Bearing this in mind, a comparison of results for the diquark gap as a function of the quark chemical potential can nevertheless be valuable to gain at least a qualitative understanding of the underlying dynamics.

To be specific, let us compare our present results for the diquark gap obtained from the computation with "gapped" gluons with those from one of the early seminal model studies in this field [48] and our recent results [28], see orange band in Fig. 2. ${ }^{12}$ The width of this band represents an estimate for the theoretical uncertainty in this study. The calculations reported in Ref. [28] are also based on an

\footnotetext{
${ }^{11}$ In practice, the scale $\bar{k}$ should come with an implicit dependence on the chemical potential which, however, is expected to be weak for sufficiently small values of the chemical potential.

${ }^{12}$ Here, we restrict ourselves to the case with "gapped" gluons since gluonic contributions are expected to be (partially) suppressed in the low-energy regime as a consequence of the Anderson-Higgs mechanism anyhow.
} 
analysis of RG flows of dense QCD matter starting from the underlying quark-gluon dynamics. Compared with our present work, however, a Fierz-complete ansatz for the four-quark interaction channels has been employed in Ref. [28]. Whereas this aspect appears to be less relevant when the quark chemical potential becomes large (since the diquark channel considered in the present work has been found to be most dominant in this regime [28,47]), Fierzincompleteness becomes more and more of an issue when the chemical potential is decreased, see Ref. [47] for a detailed discussion. In fact, not only the scalar-pseudoscalar interaction channel but also vector channels become relevant when we approach the nucleonic low-density regime [47], see also Refs. [119-121]. Since we have not included such channels in our present work, our results are expected to become less reliable when the chemical potential becomes small. Comparing our results for the size of the diquark gap with those from Ref. [28] (see left panel of Fig. 2), we observe that our present results exceed those from Ref. [28] for $\mu / \Lambda_{\mathrm{QCD}} \lesssim 2.1$. Therefore, we cautiously conclude that four-quark interaction channels other than the diquark channel become relevant in this regime. Note that, toward smaller chemical potentials (associated with densities $n / n_{0} \lesssim 5$, where $n_{0}$ is the nuclear saturation density), the results from the Fierz-complete study in Ref. [28] are remarkably consistent with those from low-energy models (e.g., Ref. [48]), see caption of Fig. 2 and also Ref. [28] for a discussion. Although the range of chemical potentials studied in our present work is beyond the range of values that can be reliably studied with low-energy models, we may cautiously deduce from this discussion that the inclusion of gluonic contributions leads to an increase of the diquark gap.

In the regime associated with diquark-channel dominance, the results for the diquark gap from the aforementioned Fierz-complete calculation (see Ref. [28]) and our present study are remarkably consistent. Note that, in Ref. [28], the "transition" between the high-energy degrees of freedom and the effective low-energy degrees of freedom has been performed at a fixed scale $\Lambda_{0}$. In principle, this scale should even carry a $\mu$-dependence which is however at least difficult to determine a priori. In any case, the presence of this scale introduces a systematic uncertainty in the results, as indicated by the width of the orange band. We emphasize that we have removed the dependence on the scale $\Lambda_{0}$ in our present work by implementing the dynamical hadronization technique. In the regime associated with a diquark-channel dominance, where a direct comparison of the two studies is most meaningful, we observe that the use of this technique already pays off. Indeed, the presence of the scale $\Lambda_{0}$ in Ref. [28] also limits the range of accessible quark chemical potentials, $\mu \lesssim \Lambda_{0}$. Since the transformation of high-energy degrees of freedom into low-energy degrees of freedom is performed continuously in our present work, the range of chemical potentials is only constrained by the requirement that the chemical potential should be sufficiently smaller than the initial RG scale $\Lambda$.

\section{TOWARD CONSTRAINTS FOR LOW-ENERGY MODELS OF DENSE QCD MATTER}

\section{A. Low-energy model couplings at high density}

Let us now turn to a discussion of the IR values of the quark-diquark coupling $h$ and the four-diquark coupling $\lambda_{\Delta}$ which often play an important role in the construction of low-energy models of dense QCD matter.

From the right panel of Fig. 2, we deduce that the quarkdiquark coupling and the four-diquark coupling are smaller in the approximation with "gapped" gluons in the lowenergy regime than in the approximation with ungapped gluons. However, their qualitative behavior as a function of the chemical potential is the same in the two approximations. Indeed, we observe that these two couplings decrease with increasing chemical potential in both cases. This simultaneous decrease is in accordance with our observation that the size of the gap appears to "saturate" for increasing chemical potential, as also suggested by our analytic study of the scaling behavior of the gap $\Delta_{\text {gap }} \sim k_{\mathrm{SB}}$, see Eq. (19). In fact, a decrease of the four-diquark coupling $\lambda_{\Delta}$ with increasing chemical potential tends to "pull" the position of the minimum of the effective action to larger values. This change of the position of the minimum needs to be compensated by a corresponding decrease of the quarkdiquark coupling $h$ such that the gap $\Delta_{\text {gap }}$ "saturates" for increasing chemical potential. From a phenomenological standpoint, the behavior of the quark-diquark coupling and the four-diquark coupling suggests that interactions between quarks and diquarks as well as among diquarks themselves become weaker when the density is increased, indicating that QCD is effectively described by a state of weakly coupled color-superconducting matter at (very) high densities.

Of course, the actual values of the quark-diquark coupling and the four-diquark coupling depend on the regularization scheme as specified by the regulator function in our RG flow study. However, the widths of the uncertainty bands shown in Fig. 2 are essentially determined by the variation of the strong coupling at the initial RG scale. The uncertainty arising from a variation of the regulator function is found to be much smaller, see our discussion in Appendix A for details.

\section{B. QCD-constrained low-energy model}

From the standpoint of model building, it may be beneficial to employ the results from our RG study to constrain existing low-energy models of dense QCD matter. In the following, we shall demonstrate this aspect by considering the following quark-diquark model: 


$$
\begin{aligned}
S_{\mathrm{LEM}}= & \int \mathrm{d}^{4} x\left\{\bar{\psi}_{a}\left(\mathrm{i} \not \partial-\mathrm{i} \mu \gamma_{0}\right) \psi_{a}+\frac{1}{2} \bar{\lambda}_{\mathrm{csc}}^{-1} \bar{\Delta}_{a}^{*} \bar{\Delta}_{a}\right. \\
& +\frac{\bar{\lambda}_{\Delta}}{\bar{h}^{4}}\left(\bar{\Delta}_{a}^{*} \bar{\Delta}_{a}\right)^{2}+\frac{1}{2} \mathrm{i}\left(\psi_{b}^{T} \mathcal{C} \gamma_{5} \tau_{2} \bar{\Delta}_{a} \epsilon_{a b c} \psi_{c}\right) \\
& \left.-\frac{1}{2} \mathrm{i}\left(\bar{\psi}_{b} \gamma_{5} \tau_{2} \bar{\Delta}_{a}^{*} \epsilon_{a b c} \mathcal{C} \bar{\psi}_{c}^{T}\right)\right\},
\end{aligned}
$$

where $a, b, c$ are color indices and we have suppressed flavor indices for readability. The action $S_{\text {LEM }}$ basically represents a frequently employed low-energy model of dense QCD matter (for reviews, see Refs. [2-6]), except for the fact that we also allow for a four-diquark coupling. The inclusion of the latter is inspired by our RG study which suggests that four-diquark interactions are generated dynamically already at high scales. Therefore, such interactions should be expected to be present at scales of the order of the "hadronic" scale $\Lambda_{\text {LEM }} \sim \mathcal{O}(1 \mathrm{GeV})$ at which low-energy models are usually defined.

In the action $S_{\text {LEM }}$ defining our model, we have introduced the fields $\bar{\Delta}_{a}$ which are directly related to the diquark fields $\Delta_{a}$ in the ansatz (3) for the effective action underlying our fRG study. We have $\bar{\Delta}_{a}=\bar{h} \Delta_{a}$. Since we shall assume that the quark-diquark coupling $\bar{h}$ in our model (20) does not depend on the RG scale $k$, it is indeed convenient to rescale the original diquark fields in this way. In fact, Yukawa-type couplings such as the quark-diquark coupling are often treated as scale-independent quantities in low-energy model studies. In any case, the introduction of the fields $\bar{\Delta}_{a}$ allows us to identify the coefficient of the curvature term $\sim \bar{\Delta}_{a}^{*} \bar{\Delta}_{a}$ in Eq. (20) with the inverse of the four-quark coupling $\bar{\lambda}_{\text {csc }}$ (up to a numerical factor), see our discussion of the relation of the curvature and the fourquark coupling in Sec. III C. Note that, by comparing the ansatz (3) for the effective action underlying our fRG study with the action $S_{\text {LEM }}$ of our low-energy model, we observe that the effective action (3) encompasses the action $S_{\text {LEM }}$.

From a computation of the effective action $\Gamma_{\text {LEM }}$ associated with the action $S_{\mathrm{LEM}}$, we can in principle extract thermodynamic quantities which are relevant for phenomenological applications. However, this requires to fix the parameters of the model in the first place. In the following, we shall illustrate how this can be done in a mean-field study of $\Gamma_{\mathrm{LEM}}$. The derivation of the corresponding effective action can be found in Appendix C.

Let us start our discussion of the determination of the model parameters by considering the four-quark coupling $\bar{\lambda}_{\text {csc }}$ in Eq. (20). Our analytic study of the four-quark coupling in Sec. III C [in particular, see the discussion of Eqs. (15)-(19)] suggests that this coupling depends only weakly on the chemical potential, provided that we consider RG scales which are sufficiently large compared to the chemical potential. This is in accordance with our numerical results where we observe that $\bar{\lambda}_{\mathrm{csc}}$ evaluated at scales sufficiently greater than the chemical potential shows

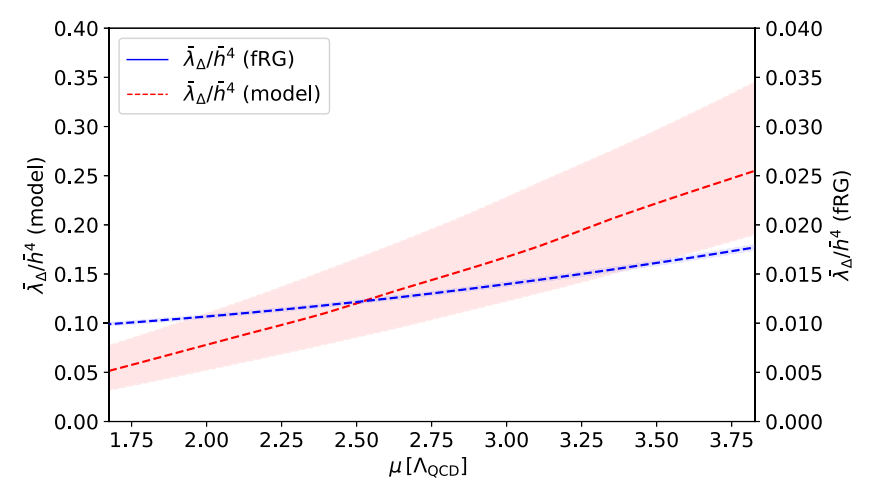

FIG. 3. Model parameter $\bar{\lambda}_{\text {eff }}=\bar{\lambda}_{\Delta} / \bar{h}^{4}$ as a function of the chemical potential compared with the RG results for $\bar{\lambda}_{\Delta} / \bar{h}^{4}$ as obtained from an evaluation of the flow at $k=\Lambda_{\mathrm{LEM}}=1 \mathrm{GeV}$. The shaded (blue) band associated with the RG results for $\bar{\lambda}_{\Delta} / \bar{h}^{4}$ reflects the uncertainty arising from a variation of the strong coupling at the initial RG scale. In case of the model parameter, the shaded (red) band results from the uncertainty band associated with our RG estimate for the gap. Note that, in our model study, we adjust the parameter $\bar{\lambda}_{\text {eff }}=\bar{\lambda}_{\Delta} / \bar{h}^{4}$ such that we recover the RG results for the gap $\Delta_{\text {gap }}$ as obtained in the approximation with "gapped" gluons in the low-energy regime.

only a very mild dependence on the chemical potential. Since we fix the model parameters at a scale $\Lambda_{\text {LEM }}>\mu$, we shall therefore assume that the parameter $\bar{\lambda}_{\text {csc }}$ does not depend on the chemical potential. However, the value of the effective four-diquark coupling $\bar{\lambda}_{\text {eff }}=\bar{\lambda}_{\Delta} / \bar{h}^{4}$ is assumed to depend on the chemical potential. The latter assumption is also in accordance with our RG results, see Fig. 3. The actual values of the model parameters $\bar{\lambda}_{\text {csc }}$ and $\bar{\lambda}_{\text {eff }}$ for a given value of the chemical potential are finally determined by tuning them such that we recover the value of the gap $\Delta_{\text {gap }}$ as obtained in our RG study. We emphasize again that we only consider $\bar{\lambda}_{\text {eff }}$ to be $\mu$-dependent. The value of the four-quark coupling $\bar{\lambda}_{\text {csc }}$ remains constant for all values of the chemical potential considered below.

In the following we choose $\Lambda_{\mathrm{LEM}}=1 \mathrm{GeV}\left(\approx 4.8 \Lambda_{\mathrm{QCD}}\right)$ which enables us to cover a reasonably large range of chemical potentials. For the four-quark coupling $\bar{\lambda}_{\text {csc }}$, we choose $\bar{\lambda}_{\text {csc }}^{-1} \approx 0.197 \mathrm{GeV}^{2}$ (for all chemical potentials considered here). For a given value of the chemical potential, the model parameter $\bar{\lambda}_{\text {eff }}$ is then determined by tuning it such that the value of the gap in our model study agrees with the one found in our RG study. Here, we focus on the results for the gap as obtained in the approximation with "gapped" gluons in the low-energy regime. However, we shall also comment on the case of ungapped gluons below.

In Fig. 3, we show the model parameter $\bar{\lambda}_{\text {eff }}$ as a function of the chemical potential. There, we also present our fRG results for this quantity as obtained from an evaluation of the RG flow at the characteristic model scale $k=\Lambda_{\text {LEM }}$. From this we deduce that the dependence of the model 
parameter on the chemical potential is compatible with our fRG results. Indeed, in both cases, we observe an increase with increasing chemical potential. Note that this is also the case when we evaluate the RG flow at lower scales. Finally, we add that a larger value of the four-quark coupling $\bar{\lambda}_{\mathrm{csc}}$ requires to choose larger values of $\bar{\lambda}_{\text {eff }}$ to ensure that the gap $\Delta_{\text {gap }}$ as a function of the chemical potential remains unchanged.

\section{Thermodynamics}

We now use our QCD-constrained model to estimate the speed of sound of dense QCD matter. To this end, we first consider the pressure $P$ as obtained from the effective action $\Gamma_{\text {LEM }}$ evaluated at the ground state $(\mathrm{gs})$ :

$$
P=-\left.\frac{1}{V_{4}} \Gamma_{\mathrm{LEM}}\right|_{\mathrm{gs}, \mu}+P_{0}
$$

Here, $V_{4}$ is the spacetime volume. The determination of the (vacuum) constant $P_{0}=\left.\left(1 / V_{4}\right) \Gamma_{\mathrm{LEM}}\right|_{\mathrm{gs}, \mu=0}$ requires to compute the ground state in the vacuum. In QCD, the ground state is governed by spontaneous chiral symmetry breaking in the low-density regime. Since we only take into account diquarklike interaction channels (which have been found to be most dominant at high densities $[28,47])$, the low-density regime is not reliably accessible in our present study. However, at higher densities, derivatives of the pressure with respect to the chemical potential are accessible. A phenomenologically relevant quantity of this kind is the speed of sound $c_{s}$ :

$$
c_{s}=\frac{1}{\sqrt{\mu}}\left(\frac{\partial P}{\partial \mu}\right)^{\frac{1}{2}}\left(\frac{\partial^{2} P}{\partial \mu \partial \mu}\right)^{-\frac{1}{2}} .
$$

By solving the baryon density $n$,

$$
n=\frac{1}{3} \frac{\partial P}{\partial \mu}
$$

for the chemical potential $\mu$, we can then compute the speed of sound as a function of the density.

In Fig. 4, we compare the speed of sound squared as a function of the density as obtained from our QCD-constrained model with results from a previous fRG study and calculations based on chiral EFT interactions at low densities. The green-shaded band associated with our model study originates from the uncertainty in the gap, see Fig. 2. Starting at high densities, we find that the speed of sound increases with decreasing density. In particular, the speed of sound is found to be greater than the one of the noninteracting quark gas in the considered density regime. Note that our present estimate for the speed of sound is in reasonable agreement with the one from Ref. [28] for $n / n_{0} \gtrsim 7$. This is essentially the density regime where the diquark interaction channel has been found to be most

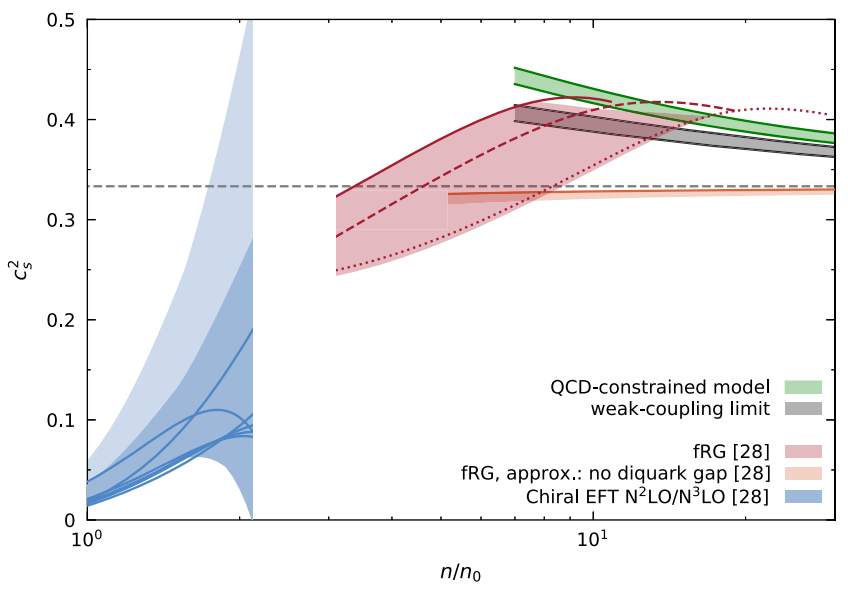

FIG. 4. Speed of sound squared (in units of the speed of light squared) as a function of the baryon density $n$ (in units of the nuclear saturation density $n_{0}$ ) as obtained from calculations based on chiral EFT (blue-shaded bands) [28], an fRG study taking into account the formation of a diquark gap (red-shaded band) [28], an fRG study based on an approximation without taking into account a diquark gap [28], and from our QCD-constrained model (green-shaded band), including the result in the weakcoupling limit (black-shaded band). The gray dashed line is associated with the result for the speed of sound squared of the noninteracting quark gas.

dominant in a Fierz-complete study $[28,47]$. For lower densities, the dynamics is governed by chiral interaction channels and therefore this regime is not accessible in our present analysis. Still, the behavior of the speed of sound at high densities observed in our present study and the one found at low(er) densities in Ref. [28] (chiral EFT and fRG) suggests the existence of a maximum in the speed of sound for $n / n_{0} \lesssim 10$. Of course, a more accurate determination of the speed of sound from the full fRG flow presented in this work - rather than from our "QCD-constrained model"-is in order and will be presented elsewhere. Based on our previous studies [28,47], such a calculation then also requires the inclusion of the chiral dynamics.

We rush to add that we have also analyzed the dependence of our results on our choice for the model parameters. Indeed, we have some freedom in the model parameters since we adjust two parameters, $\bar{\lambda}_{\text {csc }}$ and $\bar{\lambda}_{\text {eff }}$, to reproduce one quantity, namely the gap. Importantly, we find that the dependence on the actual choice for the parameters $\bar{\lambda}_{\mathrm{csc}}$ and $\bar{\lambda}_{\text {eff }}$ is only mild and does not alter the qualitative behavior of the speed of sound as a function of the density, provided that the parameters are tuned such that the gap remains unchanged as a function of the chemical potential.

A change of the size of the gap as a function of the chemical potential affects the speed of sound. For example, the model parameters can also be adjusted such that we recover the gap obtained in the fRG calculations with ungapped gluons in the low-energy regime, which is significantly greater than the one found in the 
approximation with gapped gluons (see Fig. 2). This results in an increase of the speed of sound squared of up to $70 \%$ toward the lower end of the considered density range. However, the qualitative dependence of the speed of sound (squared) as a function of the density is not altered, i.e., it still increases when the density is decreased.

The robustness of our results for the speed of sound with respect to a variation of the model parameters becomes at least plausible by considering the weak-coupling limit of the effective action which is analytically accessible. In this limit of weak four-quark and four-diquark coupling, the pressure reads $[2,77,122,123]$ :

$$
P=P_{\mathrm{SB}}\left(1+\frac{2\left|\Delta_{\text {gap }}\right|^{2}}{\mu^{2}}+\ldots\right) .
$$

Here, $P_{\mathrm{SB}}=\mu^{4} /\left(2 \pi^{2}\right)$ is the pressure of the noninteracting quark gas, ${ }^{13}$ i.e., the pressure in the so-called StefanBoltzmann (SB) limit. Interestingly, the expression (24) does not exhibit an explicit dependence on the model parameters. It only depends on the chemical potential and the gap, which is a physical observable. ${ }^{14}$ This expression may therefore be associated with regimes where $\left|\Delta_{\text {gap }}\right| / \mu$ is sufficiently small. Of course, the gap depends implicitly on the model parameters, such as the four-quark coupling, as also suggested by our analytic study of the scaling behavior of the symmetry breaking scale $k_{\mathrm{SB}}$ and the gap $\Delta_{\text {gap }} \sim k_{\mathrm{SB}}$, see Eq. (19). Moreover, we observe that the leading-order correction to the Stefan-Boltzmann limit is quadratic in $\left|\Delta_{\text {gap }}\right| / \mu$. Thus, it increases by, e.g., a factor of four when the gap is increased by a factor of two for a given chemical potential.

Plugging now our fRG results for, e.g., the gap obtained in the approximation with gapped gluons into the expression (24) for the pressure, we can estimate the speed of sound with the aid of Eq. (22). Recall that the gap in our RG study is generated from the fundamental quark-gluon dynamics and it therefore depends on the strong coupling $g$. This is made explicit in Eq. (19). In any case, reassuringly, we find again that the speed of sound exceeds the value of the noninteracting quark gas and increases when the density is decreased, see Fig. 4. The width of the associated black-shaded band in Fig. 4 results from the

\footnotetext{
${ }^{13}$ Gluons do not contribute to $P_{\mathrm{SB}}$ in the zero-temperature limit.

${ }^{14}$ In principle, this expression is also encompassed in our present fRG study anchored in QCD since it follows from a consideration of the weak-coupling limit of the one-loop approximation of the effective action, see Ref. [77]. Recall that we have $\lambda_{\text {csc }} \sim g^{4}$ for the four-quark coupling, see Eq. (17). A detailed discussion of this aspect will be presented elsewhere. In any case, in QCD with two massless quark flavors at high density, the gap sets the scale. It is therefore reasonable to expect that the pressure in units of the pressure of the noninteracting quark gas can be expanded in powers of the dimensionless quantity $\left|\Delta_{\text {gap }}\right| / \mu$.
}

width of the band of the gap shown in Fig. 2. Note that we have $0.3 \lesssim\left|\Delta_{\text {gap }}\right| / \mu \lesssim 0.6$ in the considered density range.

It is also worth adding that the observed behavior of the speed of sound as a function of the density has not been observed in fRG calculations which do not take into account the formation of a gap at high densities, see Ref. [28] and Fig. 4 for an illustration.

In accordance with Ref. [28], we therefore cautiously conclude from our analysis that the appearance of a maximum in the speed of sound-which exceeds the value of the noninteracting quark gas-appears to be tightly connected to the formation of a diquark gap. Our present analysis suggests that the maximum appears in the regime $n / n_{0} \lesssim 10$ for isospin-balanced QCD matter, although the determination of its exact position requires additional more advanced studies, as already indicated above. With respect to astrophysical applications, it is still worth mentioning that the analysis of constraints from neutron-star masses also strongly suggests the existence of a maximum in the speed of sound for neutron-rich matter [24,57-60]. In any case, our present findings may already provide useful information for future studies of thermodynamic quantities at supranuclear densities and also for the further development of existing models of dense QCD matter.

\section{CONCLUSIONS}

Starting from the fundamental quark and gluon degrees of freedom in the high-energy regime, we have studied the dynamical formation of diquarks in the low-energy regime at high densities, with the strong coupling at the initial RG scale as the only input parameter. With the present work, we have therefore laid the methodological foundation which will enable us to provide updates of our recent computation of the EOS of dense QCD matter [28]. In particular, we have successfully demonstrated that the dynamical hadronization technique allows us to remove the dependence of an auxiliary scale $\Lambda_{0}$ used in Ref. [28] to parametrize the "transition" between the (effective) degrees of freedom at high and low energies. Moreover, this technique allows us to extend our studies to (very) high densities, even beyond the densities discussed in Ref. [28]. As a first application, we computed the diquark gap over a wide range of chemical potentials. We also combined these methodological advances with the implementation of a recently developed class of regulators, which is well suited for studies of relativistic theories in the presence of a Cooper instability [78].

The comparison of our present work with our previous studies [28,47] turned out to be very beneficial, also for future computations of the EOS of dense matter. For example, approaching the nucleonic low-density regime from high densities (associated with large quark chemical potentials), this comparison indicates that the use of a Fierz-complete basis of (gluon-induced) four-quark interactions becomes more and more relevant. At high densities, 
where the diquark interaction channel is most dominant, a Fierz-incomplete ansatz including only the diquark channel in the matter sector appears to be a reasonable approximation in terms of the number of included quark interaction channels.

Our study of the RG flow of dense QCD matter allowed us to analyze the dependence of the size of the diquark gap on the strong coupling and the quark chemical potential. Moreover, we have discussed that the inclusion of gluon screening effects in our calculations may become particularly relevant at (very) high densities. We argued that such effects may even lead to a decrease of the symmetry breaking scale and the diquark gap for some intermediate range of the chemical potential, before they eventually increase again $[6,43]$. In any case, it appears reasonable to expect that the inclusion of gluon screening effects in our calculations will render the symmetry breaking scale and the diquark gap smaller. However, these effects should become subleading when the chemical potential is decreased.

In addition to gluon screening effects, the inclusion of fluctuations of the diquark fields is important. Above the symmetry breaking scale, this is straightforward but is expected to be subleading anyhow. In fact, the corresponding contributions are parametrically suppressed by large screening masses of the diquarks in this regime. Below the symmetry breaking scale, however, the situation is more involved. Here, the inclusion of diquark fluctuations requires to deal with an Anderson-Higgs-type mechanism in future studies, which eventually leads to a suppression of gluonic contributions to the RG flow.

Finally, we add that we have demonstrated how our present fRG study may already be used to further develop existing models of dense QCD matter. Based on this, we have presented an analysis of a quantity which is of great interest for phenomenological applications, namely the speed of sound. Starting at high densities, our results indicate an increase of this quantity when the density is decreased, suggesting the existence of a maximum in the speed of sound of isospin-balanced QCD matter at supranuclear densities. This maximum would exceed the asymptotic value of the speed of sound associated with the limit of a noninteracting quark gas. Moreover, our study indicates that the actual height of this maximum may be sensitive to the actual size of the gap in the fermionic excitation spectrum. These observations may also be interesting with respect to astrophysical applications where the equation of state of QCD matter enters as an input. Note that the existence of a maximum in the speed of sound of neutronrich matter is strongly supported by the analysis of constraints from neutron-star masses [24,57-60].

It is clear that our present study can and should be improved in various directions. Still, we believe that it already provides an important insight into the dynamics of dense QCD matter. Very importantly, our present work sets the methodological stage that allows us to connect the perturbative high-energy regime associated with quarks and gluons with the nonperturbative low-energy regime governed by the emergence of (color-)superconducting ground states at high densities. By successively implementing the aforementioned extensions in our present study, we expect that it will be possible to systematically improve our recent prediction for the EOS of nuclear matter over a wide range of densities [28].

\section{ACKNOWLEDGMENTS}

The authors would like to thank A. Geißel, K. Hebeler, M. Leonhardt, and J. M. Pawlowski for useful discussions and comments on the manuscript. Moreover, J. B. acknowledges useful discussions with J. Berges. As members of the fQCD collaboration [124], the authors also would like to thank the other members of this collaboration for discussions and providing data for cross-checks. J. B. acknowledges support by the DFG under Grants No. BR 4005/4-1 and No. BR 4005/6-1 (Heisenberg program). This work is supported by the Deutsche Forschungsgemeinschaft (DFG, German Research Foundation)—Projektnummer 279384907-SFB 1245 and by the State of Hesse within the Research Cluster ELEMENTS (Project No. 500/10.006).

\section{APPENDIX A: THRESHOLD FUNCTIONS AND REGULATOR}

In this Appendix, we list the so-called threshold functions which appear in our RG flow equations and correspond to 1PI Feynman diagrams. These functions also encode the regularization scheme dependence. The regularization scheme is determined by so-called regulator functions for the fermionic and bosonic fields, respectively. These functions are constructed such that they suitably modify the dispersion relation of the associated particles for any finite $k$ and disappear in the limit $k \rightarrow 0$.

In our present work, we employ so-called spatial regulators and integrate out fermionic fluctuations around the Fermi surface which is suitable in the presence of a Cooper instability, see Ref. [78] for a detailed discussion. To this end, it is convenient to introduce quasiparticle dispersion relations for the fermions:

$$
\epsilon_{ \pm}=(\mu \pm|\vec{p}|)\left(1+r_{ \pm}\right) .
$$

These relations depend on the regularization scheme which, in our case, is specified by the following so-called fermionic regulator-shape functions:

$$
r_{ \pm}:=r_{\psi}\left(x_{ \pm}\right),
$$

where $x_{ \pm}=(\mu \pm|\vec{p}|)^{2} / k^{2}$. For convenience, we have used shape functions of the following form: 


$$
r_{\psi}\left(x_{ \pm}\right)=-1+\frac{1}{\sqrt{1-\left(\sum_{n=0}^{N} \frac{1}{n !} x_{ \pm}^{n}\right)^{-1}}}
$$

These functions cut off the spatial momenta exponentially in the limit $N \rightarrow \infty$. To be specific, we have used $N=$ $4,6,8$ in our numerical calculations to analyze the regularization-scheme dependence of our results.
For the gauge fields, we have employed the corresponding bosonic version of this class of regulators:

$$
r_{A}(x)=\frac{1}{\sum_{n=1}^{N} \frac{1}{n !} x^{n}}
$$

where $x=\vec{p}^{2} / k^{2}$. In this work, we have used $N=4,6,8$ as for the fermionic regulator.

With these definitions at hand, let us now define the threshold functions entering our RG flow equations:

$$
\begin{aligned}
b_{(0,4)}^{(1)}(\tilde{\mu}, \tilde{\chi})= & \int_{-\infty}^{\infty} \frac{\mathrm{d} y_{0}}{2 \pi} \int \frac{\mathrm{d}^{3} y}{(2 \pi)^{3}} \tilde{\partial}_{t}\left\{\tilde { G } _ { A } ^ { 2 } \tilde { G } _ { + } \tilde { G } _ { - } \left(1-\frac{29}{40} \tilde{\chi}\left(\frac{1}{y_{0}^{2}+\tilde{\epsilon}_{-}^{2}+\tilde{\chi}}+\frac{1}{y_{0}^{2}+\tilde{\epsilon}_{+}^{2}+\tilde{\chi}}\right)\right.\right. \\
& \left.\left.+\frac{3}{4} \tilde{\chi}^{2} \frac{1}{\left(y_{0}^{2}+\tilde{\epsilon}_{+}^{2}+\tilde{\chi}\right)} \frac{1}{\left(y_{0}^{2}+\tilde{\epsilon}_{-}^{2}+\tilde{\chi}\right)}\right)\right\},
\end{aligned}
$$

where $y_{0}=p_{0} / k, \vec{y}=\vec{p} / k, \tilde{\epsilon}_{ \pm}=\epsilon_{ \pm} / k$, and $\tilde{\chi}$ is a parameter associated with the diquark gap in our case. Note that $\vec{y}^{2}=x$. Moreover, we have introduced the operator $\tilde{\partial}_{t}$ :

$$
\tilde{\partial}_{t}=\left(\partial_{t} r_{A}-\eta_{A} r_{A}\right) \partial_{r_{A}}+\left(\partial_{t} r_{+}\right) \partial_{r_{+}}+\left(\partial_{t} r_{-}\right) \partial_{r_{-}}
$$

The functions $\tilde{G}_{A}$ and $\tilde{G}_{ \pm}$are defined as

$$
\tilde{G}_{A}=\frac{1}{y_{0}^{2}+\vec{y}^{2}\left(1+r_{A}\right)} \quad \text { and } \quad \tilde{G}_{ \pm}=-\frac{1}{y_{0}+\mathrm{i} \tilde{\epsilon}_{ \pm}}
$$

respectively. In addition to the threshold function defined in Eq. (A5), the following threshold functions appear in our RG flow equations for the curvature $\epsilon_{\mu}$, the diquark condensate $\kappa$, the quark-diquark coupling $h$, and the four-diquark coupling $\lambda_{\Delta}$ :

$$
\begin{gathered}
b_{(0,4)}^{(2)}(\tilde{\mu}, \tilde{\chi})=\frac{1}{2} \int_{-\infty}^{\infty} \frac{\mathrm{d} y_{0}}{2 \pi} \int \frac{\mathrm{d}^{3} y}{(2 \pi)^{3}} \tilde{\partial}_{t}\left\{\tilde{G}_{A}^{2}\left(\frac{1}{y_{0}^{2}+\tilde{\epsilon}_{+}^{2}+\tilde{\chi}}+\frac{1}{y_{0}^{2}+\tilde{\epsilon}_{-}^{2}+\tilde{\chi}}\right)\right\}, \\
b_{(0,4)}^{(3)}(\tilde{\mu}, \tilde{\chi})=\int_{-\infty}^{\infty} \frac{\mathrm{d} y_{0}}{2 \pi} \int \frac{\mathrm{d}^{3} y}{(2 \pi)^{3}} \tilde{\partial}_{t}\left\{\tilde{G}_{A}^{2}\left(\frac{1}{\left(y_{0}^{2}+\tilde{\epsilon}_{-}^{2}+\tilde{\chi}\right)^{2}}+6 \frac{1}{\left(y_{0}^{2}+\tilde{\epsilon}_{-}^{2}+\tilde{\chi}\right)} \frac{1}{\left(y_{0}^{2}+\tilde{\epsilon}_{+}^{2}+\tilde{\chi}\right)}+\frac{1}{\left(y_{0}^{2}+\tilde{\epsilon}_{+}^{2}+\tilde{\chi}\right)^{2}}\right) \tilde{\chi}\right\}, \\
b_{(0,4)}^{(A)}(\tilde{\mu}, \tilde{\chi})=\frac{5}{36} b_{(0,4)}^{(1)}(\tilde{\mu}, \tilde{\chi})+\frac{4}{9} b_{(0,4)}^{(2)}(\tilde{\mu}, \tilde{\chi})+\frac{5}{48} b_{(0,4)}^{(3)}(\tilde{\mu}, \tilde{\chi}), \\
b_{(1,2)}^{(A)}(\tilde{\mu}, \tilde{\chi})=\frac{1}{2} \int_{-\infty}^{\infty} \frac{\mathrm{d} y_{0}}{2 \pi} \int \frac{\mathrm{d}^{3} y}{(2 \pi)^{3}} \tilde{\partial}_{t}\left\{\tilde{G}_{A}\left(\frac{1}{y_{0}^{2}+\tilde{\epsilon}_{+}^{2}+\tilde{\chi}}+\frac{1}{y_{0}^{2}+\tilde{\epsilon}_{-}^{2}+\tilde{\chi}}\right)\right\}, \\
b_{(4,0)}(\tilde{\mu}, \tilde{\chi})=\frac{1}{2} \int_{-\infty}^{\infty} \frac{\mathrm{d} y_{0}}{2 \pi} \int \frac{\mathrm{d}^{3} y}{(2 \pi)^{3}} \tilde{\partial}_{t}\left\{\frac{1}{\left(y_{0}^{2}+\tilde{\epsilon}_{-}^{2}+\tilde{\chi}\right)^{2}}+\frac{1}{\left(y_{0}^{2}+\tilde{\epsilon}_{+}^{2}+\tilde{\chi}\right)^{2}}\right\}, \\
b_{(2,0)}(\tilde{\mu}, \tilde{\chi})=\frac{1}{2} \int_{-\infty}^{\infty} \frac{\mathrm{d} y_{0}}{2 \pi} \int \frac{\mathrm{d}^{3} y}{(2 \pi)^{3}} \tilde{\partial}_{t}\left\{\frac{1}{y_{0}^{2}+\tilde{\epsilon}_{-}^{2}+\tilde{\chi}}+\frac{1}{y_{0}^{2}+\tilde{\epsilon}_{+}^{2}+\tilde{\chi}}\right\} .
\end{gathered}
$$

The threshold function appearing in the anomalous dimension of the diquark field is defined as follows: 
$d_{(2,0)}(\tilde{\mu}, \tilde{\chi})=\frac{1}{2} \int_{-\infty}^{\infty} \frac{\mathrm{d} y_{0}}{2 \pi} \int \frac{\mathrm{d}^{3} y}{(2 \pi)^{3}} \tilde{\partial}_{t}\left\{\frac{2 y_{0}^{2}}{\left(y_{0}^{2}+\tilde{\epsilon}_{ \pm}^{2}+\tilde{\chi}\right)^{3}}+\frac{1}{2\left(y_{0}-\mathrm{i} \tilde{\epsilon}_{ \pm}\right)^{2}\left(y_{0}^{2}+\tilde{\epsilon}_{ \pm}^{2}+\tilde{\chi}\right)}-\frac{3 y_{0}+\mathrm{i} \tilde{\epsilon}_{ \pm}}{2\left(y_{0}+\mathrm{i} \tilde{\epsilon}_{ \pm}\right)\left(y_{0}^{2}+\tilde{\epsilon}_{ \pm}^{2}+\tilde{\chi}\right)^{2}}\right\}$.

Note that we have defined the anomalous dimension of the diquark field via the second derivative of the corresponding loop diagram with respect to the zeroth component of the external four-momentum. Alternatively, we could have defined it via the second derivative with respect to the external spatial momentum. In the presence of a finite chemical potential, it is in principle necessary to take into account the wave function renormalization factors resulting from both definitions since the chemical potential distinguishes the zeroth component of the four-momentum from the spatial components. Nevertheless, for $k \gg \mu$, the running obtained from the two definitions should eventually be the same. However, a residual difference in the running of the two wave function renormalizations remains even for $k \gg \mu$ when a three-dimensional regulator is employed, as it is the case in this work. This difference can be traced back to the fact that three-dimensional regulators break Lorentz invariance, see Ref. [125]. In the present study, we do not aim at resolving these issues. In fact, as mentioned above, we only consider the wave function renormalization factor obtained from taking derivatives with respect to the zeroth component of the external four-momentum since it is the one associated with the direction in momentum space distinguished by the chemical potential. In addition, from a practical point of view, it should be noted that the computation of this wave function renormalization is simplified by the fact that three-dimensional regulators do not depend on the zeroth component of the four-momentum.

Finally, we define the threshold function associated with the quark contribution $\eta_{q}$ to the anomalous dimension $Z_{A}$ of the gauge fields, see Eq. (14). To this end, we first consider the RG flow equation for $Z_{A}$ :

$$
\partial_{t} Z_{A}=\left.\frac{1}{3} \frac{\partial}{\partial Q_{0}^{2}} P_{T}^{\alpha \beta}(Q) \frac{\delta_{c d}}{N_{\mathrm{c}}^{2}-1} \frac{1}{(2 \pi)^{4} \delta^{(4)}(0)} \frac{\delta}{\delta A_{\alpha}^{c}(Q)} \frac{\delta}{\delta A_{\beta}^{d}(-Q)} \partial_{t} \Gamma_{k}\right|_{Q_{0}=0, \vec{Q}=0, \Delta_{a}=\Delta_{0} \delta_{a, 3}}
$$

Here, $\Delta_{0}$ is the diquark condensate, $Q_{0}$ and $\vec{Q}$ are external momenta, and $P_{T}^{\alpha \beta}(Q)$ is the standard transversal projector. Note that the condensate $\Delta_{0}$ distinguishes a direction in color space and therefore the wave function renormalization $Z_{A}$ is in principle no longer uniform in color space. In our calculations, we have not resolved the different directions in the low-energy regime (i.e., in the presence of a condensate) since a careful analysis of this aspect requires to deal with the Anderson-Higgs mechanism. This is beyond the scope of this work. Below the symmetry breaking scale, our present definition of $Z_{A}$ as given by Eq. (A15) rather "averages" over all directions in color space. In any case, the threshold function associated with Eq. (14) is defined as

$$
d_{(2,0)}^{(A)}(\tilde{\mu}, \tilde{\chi})=-\left.\frac{4}{\bar{g}^{2}} \partial_{t} Z_{A}\right|_{\text {quark loop }},
$$

where $\tilde{\chi}=\bar{h}^{2} \Delta_{0}^{2} / k^{2}$. It is worthwhile to add that this threshold function can be written in a compact form in the symmetric regime $(\tilde{\chi}=0)$ :

$d_{(2,0)}^{(A)}(\tilde{\mu}, 0)=\frac{32}{3} \int_{-\infty}^{\infty} \frac{\mathrm{d} y_{0}}{2 \pi} \int \frac{\mathrm{d}^{3} y}{(2 \pi)^{3}} \tilde{\partial}_{t}\left\{\tilde{G}_{+}\left(\tilde{G}_{-}\right)^{3}\right\}$.

For $\tilde{\mu}=0$, we find $d_{(2,0)}^{(A)}(0,0)=2 /\left(3 \pi^{2}\right)$. By plugging this into Eq. (14), we recover the one-loop result for the quark contribution to the running of the strong coupling in case of two massless quark flavors, as it should be.

We close this Appendix on the threshold functions and the regulator by noting that the uncertainty bands given in Fig. 2 include the variation of our results arising from a variation of the regulator as parametrized by the value of $N$, see Eqs. (A3) and (A4). However, we observe that the dependence on the regulator (scheme) is much smaller than the one introduced by the variation of the initial value of the strong coupling. In the case of gapped gluons in the lowenergy regime, the weak regulator dependence is illustrated
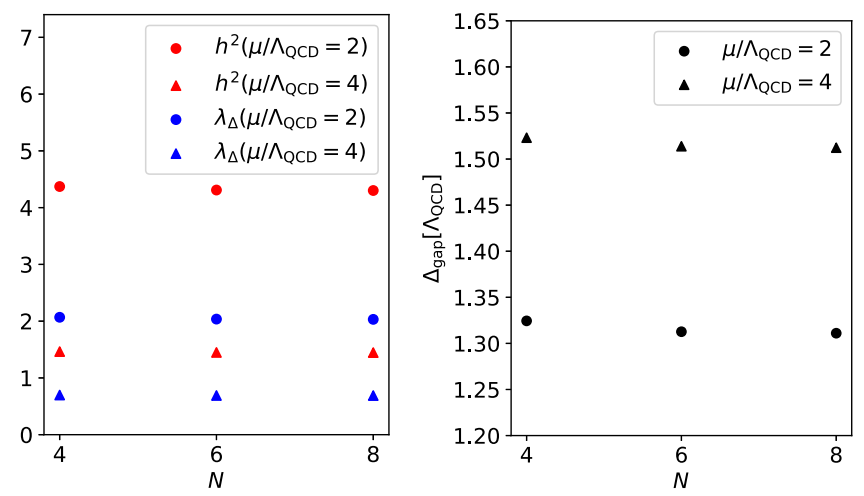

FIG. 5. Regulator dependence of the IR values of the quarkdiquark coupling $h$, the four-diquark coupling $\lambda_{\Delta}$, and the gap $\Delta_{\text {gap }}$, as specified by the parameter $N$ for two values of the chemical potential, see Eqs. (A3) and (A4). 
in Fig. 5 for the IR values of the Yukawa coupling, the four-diquark coupling, and the gap for $\mu / \Lambda_{\mathrm{QCD}}=2.0$ and $\mu / \Lambda_{\mathrm{QCD}}=4.0$.

\section{APPENDIX B: DYNAMICAL HADRONIZATION}

In this work, we employ the so-called dynamical hadronization technique to study the RG flow from the perturbative high-energy limit down to the low-energy regime which may be conveniently described by effective degrees of freedom, such as pions at low density and diquarks at high density.
Loosely speaking, we use this technique to implement continuous Hubbard-Stratonovich transformations in the RG flow which map quark selfinteraction channels onto diquark interaction channels as well as quark-diquark channels. For example, this allows us to conveniently resolve momentum dependences of, e.g., four-quark interactions and to compute the order-parameter potential. In general, this technique is even more powerful as it relies on the idea of introducing scale-dependent fields [68-74].

In the present work, we introduce $k$-dependent diquark fields, $\Delta_{a} \rightarrow \Delta_{a, k}$. The original Wetterich equation [61] is then modified as follows:

$$
\partial_{t} \Gamma_{k}=\left.\partial_{t} \Gamma_{k}\right|_{\Delta_{a, k}, \Delta_{a, k}^{*}}+\int_{p^{\prime}}\left\{\frac{\delta \Gamma_{k}}{\delta \Delta_{a, k}\left(p^{\prime}\right)} \partial_{t} \Delta_{a, k}\left(p^{\prime}\right)+\frac{\delta \Gamma_{k}}{\delta \Delta_{a, k}^{*}\left(p^{\prime}\right)} \partial_{t} \Delta_{a, k}^{*}\left(p^{\prime}\right)\right\} .
$$

The first term on the right-hand side of this equation is nothing but the original Wetterich equation evaluated on the scaledependent diquark fields. For the parametrization of the scale dependence of these fields, we make the following ansatz:

$$
\begin{aligned}
\partial_{t} \Delta_{a, k}\left(p^{\prime}\right) & =-\int \frac{\mathrm{d}^{4} p}{(2 \pi)^{4}} \int \frac{\mathrm{d}^{4} q}{(2 \pi)^{4}} \frac{\mathrm{i}}{2}\left(\bar{\psi}_{b}(p) \gamma_{5} \tau_{2} \epsilon_{a b c} \mathcal{C} \bar{\psi}_{c}^{T}(q)\right)(2 \pi)^{4} \delta^{(4)}\left(p^{\prime}-p-q\right) \partial_{t} \rho_{k}\left(p^{\prime}\right)+\Delta_{a, k}\left(p^{\prime}\right) \partial_{t} \beta_{k}\left(p^{\prime}\right), \\
\partial_{t} \Delta_{a, k}^{*}\left(p^{\prime}\right) & =\int \frac{\mathrm{d}^{4} p}{(2 \pi)^{4}} \int \frac{\mathrm{d}^{4} q}{(2 \pi)^{4}} \frac{\mathrm{i}}{2}\left(\psi_{b}^{T}(p) \mathcal{C} \gamma_{5} \tau_{2} \epsilon_{a b c} \psi_{c}(q)\right)(2 \pi)^{4} \delta^{(4)}\left(p^{\prime}-p-q\right) \partial_{t} \rho_{k}\left(p^{\prime}\right)+\Delta_{a, k}^{*}\left(p^{\prime}\right) \partial_{t} \beta_{k}\left(p^{\prime}\right),
\end{aligned}
$$

where $p=\left\{p_{0}, \vec{p}\right\}$ and correspondingly for $p^{\prime}$ and $q$. The functions $\beta_{k}$ and $\rho_{k}$ are at our disposal. In the following, we shall determine them such that quark self-interactions associated with the diquark channel are mapped onto diquark interaction channels and quark-diquark interaction channels, as it is usually done by a Hubbard-Stratonovich transformation of the quark bilinears appearing on the right-hand sides of Eqs. (B2) and (B3). However, as we allow for a scale dependence in our parametrization, we perform such a transformation continuously as a function of the RG scale $k$. This is important as four-quark interactions usually removed by such a Hubbard-Stratonovich transformation at a given scale may be regenerated in the RG flow because of processes associated with, e.g., two-gluon exchange diagrams.

For the chiral regime at low densities, the determination of the functions $\beta_{k}$ and $\rho_{k}$ has been discussed in Refs. $[68,69,71,73]$. Similar to these studies, we determine these functions by requiring that

(i) the RG flow equation of the four-quark coupling vanishes identically on all scales $k$,

(ii) the quark-diquark coupling $\bar{h}$ is momentumindependent,

(iii) $\partial_{t} Z_{\Delta}\left(p_{0}=0,|\vec{p}|=k\right)=-\eta_{\Delta} Z_{\Delta}$.

Our initial condition for the four-quark coupling, $\bar{\lambda}_{\mathrm{csc}} \rightarrow 0$ for $k \rightarrow \Lambda$ (see Sec. III A), together with the requirement (i) ensures that a four-quark interaction channel (as associated with the diquark channel) is not generated in the RG flow. The contributions to this four-quark interaction generated in the RG flow are mapped onto the scalar sector, in the spirit of a Hubbard-Stratonovich transformation. The requirement (ii) ensures that the diquark gap generated in the low-energy regime is also momentumindependent. Finally, our third requirement renders our approximation of a momentum-independent $Z_{\Delta}$-factor selfconsistent.

By plugging our ansatz (3) for the effective action together with our ansätze (B2) and (B3) for the diquark fields into the flow equation (B1) and then applying the aforementioned three requirements, we obtain the following equations for $\beta_{k}$ and $\rho_{k}$ in the symmetric regime:

$$
\begin{aligned}
\partial_{t} \beta_{k}(p)= & -\frac{Z_{\Delta} p^{2}+4 \mathrm{i} Z_{\Delta} \mu p_{0}+\Delta \bar{m}^{2}}{\bar{h}^{2}} \partial_{t} \bar{\lambda}_{\mathrm{csc}}(p) \\
& +\frac{1}{Z_{\Delta} k^{2} \bar{h}^{2}}\left[\left(Z_{\Delta} k^{2}+\Delta \bar{m}^{2}\right)^{2} \partial_{t} \bar{\lambda}_{\mathrm{csc}}(0,|\vec{p}|=k)\right. \\
& \left.-\left(\Delta \bar{m}^{2}\right)^{2}\left(\partial_{t} \bar{\lambda}_{\mathrm{csc}}(0,|\vec{p}|=k)-\partial_{t} \Delta \bar{\lambda}_{\mathrm{csc}}\right)\right],
\end{aligned}
$$

where $\Delta \bar{m}^{2}=\bar{m}^{2}-4 Z_{\Delta} \mu^{2}$, and

$$
\partial_{t} \rho_{k}(p)=\left.\frac{1}{\bar{h}} \partial_{t} \bar{\lambda}_{\mathrm{csc}}(p)\right|_{\Delta_{k}, \Delta_{k}^{*}}
$$


The quantity $\Delta \bar{\lambda}_{\text {csc }}$ is the difference of $\bar{\lambda}_{\text {csc }}(0,|\vec{p}|=k)$ and $\bar{\lambda}_{\text {csc }}(0,|\vec{p}|=0)$. In our study, we set this quantity to zero. This approximation has been discussed in Refs. [68,69]. There, it has been found that quantities such as the symmetry breaking scale and the condensate are only weakly affected by this simplification. Essentially, it only affects the position of (pseudo-)fixed points of the couplings in the symmetric regime but not their existence.

In the low-energy regime, associated with a nontrivial ground state, the equation for $\rho_{k}$ remains unchanged. However, the equation for $\beta_{k}$ changes and reads

$\partial_{t} \beta_{k}(p)=-\frac{Z_{\Delta} p^{2}+4 \mathrm{i} Z_{\Delta} \mu p_{0}}{\bar{h}^{2}} \partial_{t} \bar{\lambda}_{\mathrm{csc}}(p)+\frac{Z_{\Delta} k^{2}}{\bar{h}^{2}} \partial_{t} \bar{\lambda}_{\mathrm{csc}}(k)$.

With these equations for $\beta_{k}$ and $\rho_{k}$ at hand, the flow equations for the couplings presented in Sec. II B can be computed.

\section{APPENDIX C: HIGH-DENSITY LOW-ENERGY MODEL}

In this Appendix, we derive the effective action $\Gamma_{\mathrm{LEM}}$ for the model defined in Eq. (20) in Sec. IV. More specifically, we shall compute the effective action $\Gamma_{\text {LEM }}$ in a one-loop approximation where we only take into account the purely fermionic loop and set the wave function renormalizations associated with the diquark fields to zero. The wave function renormalizations of the quarks are assumed to be constant. Note that our derivation follows closely the one of a related model in Ref. [77].

The starting point is the classical action $S_{\text {LEM }}$ of our model:

$$
\begin{aligned}
S_{\mathrm{LEM}}= & \int \mathrm{d}^{4} x\left\{\bar{\psi}_{a}\left(\mathrm{i} \not \partial-\mathrm{i} \mu \gamma_{0}\right) \psi_{a}+\frac{1}{2} \bar{\lambda}_{\mathrm{csc}}^{-1} \bar{\Delta}_{a}^{*} \bar{\Delta}_{a}\right. \\
& +\frac{\bar{\lambda}_{\Delta}}{\bar{h}^{4}}\left(\bar{\Delta}_{a}^{*} \bar{\Delta}_{a}\right)^{2}+\frac{1}{2} \mathrm{i}\left(\psi_{b}^{T} \mathcal{C} \gamma_{5} \tau_{2} \bar{\Delta}_{a} \epsilon_{a b c} \psi_{c}\right) \\
& \left.-\frac{1}{2} \mathrm{i}\left(\bar{\psi}_{b} \gamma_{5} \tau_{2} \bar{\Delta}_{a}^{*} \epsilon_{a b c} \mathcal{C} \bar{\psi}_{c}^{T}\right)\right\} .
\end{aligned}
$$

Here, $a, b, c$ are color indices. The flavor indices are suppressed for readability.

Using, e.g., the Wetterich equation [61] and expanding the diquark fields about a homogeneous background, we obtain the following result for $\Gamma_{\mathrm{LEM}}$ :

$$
\frac{1}{V_{4}} \Gamma_{\mathrm{LEM}}=\frac{1}{V_{4}} \Gamma_{\mathrm{LEM}, \Lambda}-\frac{\mu^{4}}{6 \pi^{2}}-8 l_{0}\left(\Lambda,|\bar{\Delta}|^{2}\right),
$$

where $V_{4}$ is the spacetime volume and $|\bar{\Delta}|^{2}=\bar{\Delta}_{a}^{*} \bar{\Delta}_{a}$ (summation over $a$ is assumed). The contribution $\sim \mu^{4}$ in this expression for $\Gamma_{\text {LEM }}$ originates from quarks which do not couple to the diquark fields and therefore only appear as a "noninteracting contribution." The quark loop integral is parametrized by the function $l_{k}$ :

$$
\begin{aligned}
l_{k}\left(\Lambda,|\bar{\Delta}|^{2}\right)= & \frac{1}{2} \int \frac{\mathrm{d}^{3} p}{(2 \pi)^{3}} \theta\left(\Lambda^{2}-\vec{p}^{2}\right) \theta\left(\vec{p}^{2}-k^{2}\right) \\
& \times\left\{\sqrt{(|\vec{p}|+\mu)^{2}+|\bar{\Delta}|^{2}}+\sqrt{(|\vec{p}|-\mu)^{2}+|\bar{\Delta}|^{2}}\right\} .
\end{aligned}
$$

Here, we have employed a sharp cutoff/regulator as often used in model studies. The expression for this loop diagram for general three-dimensional regulators can be found in Ref. [77]. Within the present approximation, a different choice for the regulator would only change the values of the model parameters used in a concrete calculation (i.e., $\bar{\lambda}_{\text {csc }}$ and $\bar{\lambda}_{\text {eff }}=\bar{\lambda}_{\Delta} / \bar{h}^{4}$ ) and the numerical prefactors associated with the counter terms in $\Gamma_{\mathrm{LEM}, \Lambda}$, as these are schemedependent quantities.

The quantity $\Gamma_{\mathrm{LEM}, \Lambda}$ in Eq. (C2) includes two classes of terms. First, it contains terms which determine the form of the effective action $\Gamma_{\mathrm{LEM}, \Lambda_{\mathrm{LEM}}} \simeq S_{\mathrm{LEM}}$ at a given scale $\Lambda_{\text {LEM }}<\Lambda$. Second, $\Gamma_{\text {LEM }, \Lambda}$ includes counterterms which ensure that $\Gamma_{\text {LEM }}$ in Eq. (C2) is an RG-consistent effective action, i.e., $\Lambda \partial_{\Lambda} \Gamma_{\text {LEM }}=0$ for $\Lambda \rightarrow \infty$. To be specific, we have [77]:

$$
\begin{aligned}
\frac{1}{V_{4}} \Gamma_{\mathrm{LEM}, \Lambda}= & \frac{1}{2} \bar{\lambda}_{\mathrm{csc}}^{-1}|\bar{\Delta}|^{2}+\frac{\bar{\lambda}_{\Delta}}{\bar{h}^{4}}|\bar{\Delta}|^{4}+\left.8 l_{\Lambda_{\mathrm{LEM}}}\left(\Lambda,|\bar{\Delta}|^{2}\right)\right|_{\mu=0} \\
& +4 \mu^{2}\left(\left.\partial_{\mu}^{2} l_{\Lambda_{\mathrm{LEM}}}\left(\Lambda,|\bar{\Delta}|^{2}\right)\right|_{\mu=0}\right) .
\end{aligned}
$$

For $\Lambda=\Lambda_{\mathrm{LEM}}$, we find $l_{\Lambda_{\mathrm{LEM}}}\left(\Lambda,|\bar{\Delta}|^{2}\right)=0$ and we are left with $\Gamma_{\mathrm{LEM}, \Lambda_{\mathrm{LEM}}}=V_{4}\left((1 / 2) \bar{\lambda} \overline{\mathrm{csc}}^{-1}|\bar{\Delta}|^{2}+\left(\bar{\lambda}_{\Delta} / \bar{h}^{4}\right)|\bar{\Delta}|^{4}\right)$. In any case, inserting $\Gamma_{\mathrm{LEM}, \Lambda}$ into Eq. (C2), we find

$$
\Lambda \partial_{\Lambda} \Gamma_{\mathrm{LEM}}=-2 V_{4}|\bar{\Delta}|^{2} \mu^{2}\left(\frac{\mu}{\pi \Lambda}\right)^{2}+\mathcal{O}\left(1 / \Lambda^{4}\right) .
$$

Thus, our low-energy model described by the effective action $\Gamma_{\text {LEM }}$ is RG-consistent in a strict sense in the limit $\Lambda \rightarrow \infty$. In our numerical computations of thermodynamic observables discussed in Sec. IV, we have always ensured RG consistency by choosing sufficiently large values for $\Lambda$. For a detailed discussion of this aspect, we refer the reader to Ref. [77]. 
[1] D. Bailin and A. Love, Phys. Rep. 107, 325 (1984).

[2] K. Rajagopal and F. Wilczek, The Condensed matter physics of QCD, in At the Frontier of Particle Physics. Handbook of QCD. Vol. 1-3, edited by M. Shifman and B. Ioffe (World Scientific, Singapore, 2000), pp. 2061-2151.

[3] M. G. Alford, Annu. Rev. Nucl. Part. Sci. 51, 131 (2001).

[4] M. Buballa, Phys. Rep. 407, 205 (2005).

[5] I. A. Shovkovy, Found. Phys. 35, 1309 (2005).

[6] M. G. Alford, A. Schmitt, K. Rajagopal, and T. Schäfer, Rev. Mod. Phys. 80, 1455 (2008).

[7] K. Fukushima and T. Hatsuda, Rep. Prog. Phys. 74, 014001 (2011).

[8] K. Fukushima, J. Phys. G 39, 013101 (2012).

[9] R. Anglani, R. Casalbuoni, M. Ciminale, N. Ippolito, R. Gatto, M. Mannarelli, and M. Ruggieri, Rev. Mod. Phys. 86, 509 (2014).

[10] A. Schmitt, Lect. Notes Phys. 888, 1 (2015).

[11] G. Baym, T. Hatsuda, T. Kojo, P. D. Powell, Y. Song, and T. Takatsuka, Rep. Prog. Phys. 81, 056902 (2018).

[12] B. P. Abbott et al. (LIGO Scientific, Virgo Collaborations), Phys. Rev. Lett. 119, 161101 (2017).

[13] B. P. Abbott et al. (LIGO Scientific, Virgo Collaborations), Phys. Rev. X 9, 011001 (2019).

[14] A. L. Watts et al., Rev. Mod. Phys. 88, 021001 (2016).

[15] Z. Arzoumanian et al., in Space Telescopes and Instrumentation 2014: Ultraviolet to Gamma Ray, SPIE Proceedings Vol. 9144 (SPIE Press, 2014), p. 914420.

[16] K. C. Gendreau et al., in Space Telescopes and Instrumentation 2016: Ultraviolet to Gamma Ray, SPIE Proceedings Vol. 9905 (SPIE Press, 2016), p. 99051H.

[17] M. C. Miller et al., Astrophys. J. Lett. 918, L28 (2021).

[18] T. E. Riley et al., Astrophys. J. Lett. 918, L27 (2021).

[19] G. Raaijmakers, S. K. Greif, K. Hebeler, T. Hinderer, S. Nissanke, A. Schwenk, T. E. Riley, A. L. Watts, J. M. Lattimer, and W. C. G. Ho, Astrophys. J. Lett. 918, L29 (2021).

[20] P. Demorest, T. Pennucci, S. Ransom, M. Roberts, and J. Hessels, Nature (London) 467, 1081 (2010).

[21] J. Antoniadis et al., Science 340, 448 (2013).

[22] E. Fonseca et al., Astrophys. J. 832, 167 (2016).

[23] H. T. Cromartie et al., Nat. Astron. 4, 72 (2020).

[24] S. Huth, C. Wellenhofer, and A. Schwenk, Phys. Rev. C 103, 025803 (2021).

[25] P. Danielewicz, R. Lacey, and W. G. Lynch, Science 298, 1592 (2002).

[26] E. Epelbaum, H.-W. Hammer, and U.-G. Meissner, Rev. Mod. Phys. 81, 1773 (2009).

[27] K. Hebeler, J. M. Lattimer, C. J. Pethick, and A. Schwenk, Astrophys. J. 773, 11 (2013).

[28] M. Leonhardt, M. Pospiech, B. Schallmo, J. Braun, C. Drischler, K. Hebeler, and A. Schwenk, Phys. Rev. Lett. 125, 142502 (2020).

[29] K. Hebeler, Phys. Rep. 890, 1 (2021).

[30] J. Berges, D. U. Jungnickel, and C. Wetterich, Int. J. Mod. Phys. A 18, 3189 (2003).

[31] M. Drews and W. Weise, Phys. Lett. B 738, 187 (2014).

[32] M. Drews and W. Weise, Phys. Rev. C 91, 035802 (2015).

[33] R.-A. Tripolt, B.-J. Schaefer, L. von Smekal, and J. Wambach, Phys. Rev. D 97, 034022 (2018).
[34] K. Otto, M. Oertel, and B.-J. Schaefer, Phys. Rev. D 101, 103021 (2020).

[35] K. Otto, M. Oertel, and B.-J. Schaefer, Eur. Phys. J. Special Topics 229, 3629 (2020).

[36] B. A. Freedman and L. D. McLerran, Phys. Rev. D 16, 1130 (1977).

[37] B. A. Freedman and L. D. McLerran, Phys. Rev. D 16, 1169 (1977).

[38] V. Baluni, Phys. Rev. D 17, 2092 (1978).

[39] A. Kurkela, P. Romatschke, and A. Vuorinen, Phys. Rev. D 81, 105021 (2010).

[40] E. S. Fraga, A. Kurkela, and A. Vuorinen, Astrophys. J. Lett. 781, L25 (2014).

[41] E. S. Fraga, A. Kurkela, J. Schaffner-Bielich, and A. Vuorinen, Nucl. Phys. A956, 813 (2016).

[42] T. Gorda, A. Kurkela, P. Romatschke, M. Säppi, and A. Vuorinen, Phys. Rev. Lett. 121, 202701 (2018).

[43] D. T. Son, Phys. Rev. D 59, 094019 (1999).

[44] T. Schäfer and F. Wilczek, Phys. Rev. D 60, 114033 (1999).

[45] S. D. Hsu and M. Schwetz, Nucl. Phys. B572, 211 (2000).

[46] J. Braun, M. Leonhardt, and M. Pospiech, Phys. Rev. D 97, 076010 (2018).

[47] J. Braun, M. Leonhardt, and M. Pospiech, Phys. Rev. D 101, 036004 (2020).

[48] M. G. Alford, K. Rajagopal, and F. Wilczek, Phys. Lett. B 422, 247 (1998).

[49] R. Rapp, T. Schäfer, E. V. Shuryak, and M. Velkovsky, Phys. Rev. Lett. 81, 53 (1998).

[50] T. Schäfer and F. Wilczek, Phys. Lett. B 450, 325 (1999).

[51] J. Berges and K. Rajagopal, Nucl. Phys. B538, 215 (1999).

[52] R. D. Pisarski and D. H. Rischke, Phys. Rev. D 61, 051501 (2000).

[53] R. D. Pisarski and D. H. Rischke, Phys. Rev. D 61, 074017 (2000).

[54] W. E. Brown, J. T. Liu, and H.-c. Ren, Phys. Rev. D 61, 114012 (2000).

[55] N. J. Evans, J. Hormuzdiar, S. D. H. Hsu, and M. Schwetz, Nucl. Phys. B581, 391 (2000).

[56] D. K. Hong, V. A. Miransky, I. A. Shovkovy, and L. C. R. Wijewardhana, Phys. Rev. D 61, 056001 (2000); 62, 059903(E) (2000).

[57] P. Bedaque and A. W. Steiner, Phys. Rev. Lett. 114, 031103 (2015).

[58] I. Tews, J. Carlson, S. Gandolfi, and S. Reddy, Astrophys. J. 860, 149 (2018).

[59] S. K. Greif, G. Raaijmakers, K. Hebeler, A. Schwenk, and A. L. Watts, Mon. Not. R. Astron. Soc. 485, 5363 (2019).

[60] E. Annala, T. Gorda, A. Kurkela, J. Nättilä, and A. Vuorinen, Nat. Phys. 16, 907 (2020).

[61] C. Wetterich, Phys. Lett. B 301, 90 (1993).

[62] J. Braun, J. Phys. G 39, 033001 (2012).

[63] H. Gies and J. Jaeckel, Eur. Phys. J. C 46, 433 (2006).

[64] J. Braun and H. Gies, Phys. Lett. B 645, 53 (2007).

[65] J. Braun and H. Gies, J. High Energy Phys. 06 (2006) 024.

[66] M. Mitter, J. M. Pawlowski, and N. Strodthoff, Phys. Rev. D 91, 054035 (2015).

[67] A. K. Cyrol, M. Mitter, J. M. Pawlowski, and N. Strodthoff, Phys. Rev. D 97, 054006 (2018). 
[68] H. Gies and C. Wetterich, Phys. Rev. D 65, 065001 (2002).

[69] H. Gies and C. Wetterich, Phys. Rev. D 69, 025001 (2004).

[70] J. M. Pawlowski, Ann. Phys. (Amsterdam) 322, 2831 (2007).

[71] H. Gies, Lect. Notes Phys. 852, 287 (2012).

[72] S. Floerchinger and C. Wetterich, Phys. Lett. B 680, 371 (2009).

[73] J. Braun, L. Fister, J. M. Pawlowski, and F. Rennecke, Phys. Rev. D 94, 034016 (2016).

[74] W.-j. Fu, J. M. Pawlowski, and F. Rennecke, Phys. Rev. D 101, 054032 (2020).

[75] K. Fukushima, J. M. Pawlowski, and N. Strodthoff, arXiv:2103.01129.

[76] J. Braun, M. Leonhardt, and M. Pospiech, Phys. Rev. D 96, 076003 (2017).

[77] J. Braun, M. Leonhardt, and J. M. Pawlowski, SciPost Phys. 6, 056 (2019).

[78] J. Braun, T. Dörnfeld, B. Schallmo, and S. Töpfel, Phys. Rev. D 104, 096002 (2021).

[79] L. F. Abbott, Nucl. Phys. B185, 189 (1981).

[80] L. F. Abbott, Acta Phys. Pol. B 13, 33 (1982).

[81] M. Reuter and C. Wetterich, Nucl. Phys. B417, 181 (1994).

[82] M. Reuter and C. Wetterich, Phys. Rev. D 56, 7893 (1997).

[83] D. F. Litim and J. M. Pawlowski, On gauge invariant Wilsonian flows, in The Exact Renormalization Group, edited by A. Krasnitz, Y. A. Kubyshin, R. Potting, and P. Sá (World Scientific, Singapore, 1999), pp. 168-185.

[84] F. Freire, D. F. Litim, and J. M. Pawlowski, Phys. Lett. B 495, 256 (2000).

[85] D. F. Litim and J. M. Pawlowski, Phys. Lett. B 546, 279 (2002).

[86] H. Gies, Phys. Rev. D 66, 025006 (2002).

[87] J. Braun, H. Gies, and J. M. Pawlowski, Phys. Lett. B 684, 262 (2010).

[88] J. Braun, A. Eichhorn, H. Gies, and J. M. Pawlowski, Eur. Phys. J. C 70, 689 (2010).

[89] U. Reinosa, J. Serreau, M. Tissier, and N. Wschebor, Phys. Lett. B 742, 61 (2015).

[90] U. Reinosa, J. Serreau, M. Tissier, and N. Wschebor, Phys. Rev. D 91, 045035 (2015).

[91] N. Dupuis, L. Canet, A. Eichhorn, W. Metzner, J. M. Pawlowski, M. Tissier, and N. Wschebor, Phys. Rep. 910, 1 (2021).

[92] L. F. Abbott, M. T. Grisaru, and R. K. Schaefer, Nucl. Phys. B229, 372 (1983).

[93] J. M. Pawlowski, Int. J. Mod. Phys. A 16, 2105 (2001).

[94] D. F. Litim and J. M. Pawlowski, Phys. Rev. D 66, 025030 (2002).

[95] J. Braun, Eur. Phys. J. C 64, 459 (2009).

[96] F. Rennecke, Phys. Rev. D 92, 076012 (2015).
[97] S. Elitzur, Phys. Rev. D 12, 3978 (1975).

[98] P. W. Anderson, Phys. Rev. 130, 439 (1963).

[99] F. Englert and R. Brout, Phys. Rev. Lett. 13, 321 (1964).

[100] P. W. Higgs, Phys. Lett. 12, 132 (1964).

[101] P. W. Higgs, Phys. Rev. Lett. 13, 508 (1964).

[102] G. S. Guralnik, C. R. Hagen, and T. W. B. Kibble, Phys. Rev. Lett. 13, 585 (1964).

[103] L. McLerran and S. Reddy, Phys. Rev. Lett. 122, 122701 (2019).

[104] H. Gies, J. Jaeckel, and C. Wetterich, Phys. Rev. D 69, 105008 (2004).

[105] Y. Kusafuka and H. Terao, Phys. Rev. D 84, 125006 (2011).

[106] D. U. Jungnickel and C. Wetterich, Phys. Rev. D 53, 5142 (1996).

[107] J. Berges, D. U. Jungnickel, and C. Wetterich, Phys. Rev. D 59, 034010 (1999).

[108] J. Berges, N. Tetradis, and C. Wetterich, Phys. Rep. 363, 223 (2002).

[109] M. Reuter and C. Wetterich, Nucl. Phys. B391, 147 (1993).

[110] D. F. Litim, On the renormalization of the Abelian Higgs model and the phase transition of superconductors, Ph.D. thesis, Heidelberg University, 1995.

[111] H. Gies, S. Rechenberger, M. M. Scherer, and L. Zambelli, Eur. Phys. J. C 73, 2652 (2013).

[112] S. Bethke, Eur. Phys. J. C 64, 689 (2009).

[113] J. I. Kapusta, Phys. Rev. D 20, 989 (1979).

[114] T. Toimela, Int. J. Theor. Phys. 24, 901 (1985); 26, 1021(E) (1987).

[115] J. Braun, A. Geißel, and B. Schallmo (to be published).

[116] T. Schäfer, Nucl. Phys. B575, 269 (2000).

[117] N. J. Evans, S. D. H. Hsu, and M. Schwetz, Nucl. Phys. B551, 275 (1999).

[118] N. J. Evans, S. D. H. Hsu, and M. Schwetz, Phys. Lett. B 449, 281 (1999).

[119] Y. Song, G. Baym, T. Hatsuda, and T. Kojo, Phys. Rev. D 100, 034018 (2019).

[120] R. D. Pisarski, Phys. Rev. D 103, L071504 (2021).

[121] R.-A. Tripolt, C. Jung, L. von Smekal, and J. Wambach, Phys. Rev. D 104, 054005 (2021).

[122] K. Rajagopal and F. Wilczek, Phys. Rev. Lett. 86, 3492 (2001).

[123] I. A. Shovkovy and P. J. Ellis, Phys. Rev. C 66, 015802 (2002).

[124] J. Braun, Y.-r. Chen, W.-j. Fu, A. Geißel, F. Ihssen, J. Horak, C. Huang, J. M. Pawlowski, F. Rennecke, F. Sattler, B. Schallmo, C. Schneider, Y.-y. Tan, S. Töpfel, R. Wen, N. Wink, and S. Yin (fQCD Collaboration) (members as of January 2022).

[125] J. Braun, Phys. Rev. D 81, 016008 (2010). 